\title{
Bioanalysis of aminoglycosides using high-performance liquid chromatography
}

\author{
Seth K. Amponsah ${ }^{1}$, Joseph A. Boadu ${ }^{2}$, Daniel K. Dwamena ${ }^{1}$, Kwabena F. M. Opuni ${ }^{2,}$ \\ ${ }^{1}$ Department of Medical Pharmacology, University of Ghana Medical School, University of Ghana, Ghana \\ ${ }^{2}$ Department of Pharmaceutical Chemistry, School of Pharmacy, University of Ghana, Ghana
}

*Corresponding Author: E-mail: kfopuni@ug.edu.gh; Tel.: +233208260595

Received: November 19, 2021; Revised: December 30, 2021; Published: January 11, 2022

\begin{abstract}
Aminoglycosides are broad-spectrum antibiotics used in the treatment of gram-negative bacterial infections. Due to their nephrotoxic and ototoxic potential (narrow therapeutic index), the use of aminoglycoside for clinical indications requires monitoring. The objective of this review was to identify relevant literature reporting liquid chromatographic methods for the bioanalysis of aminoglycosides in both preclinical and clinical settings/experiments. Data on liquid chromatographic methods were collected from articles in an online academic database (PubMed, Science Direct, Scopus, and Google Scholar). All 71 articles published from 1977 to 2020 were included in the review. Reversed-phase liquid chromatography was the most used method for the bioanalysis of aminoglycosides. Fluorescence or ultraviolet detection methods were mostly used from 1977 to 2002 (51 articles), while mass spectrometry was predominantly used as a detector from 2003 to 2020 (15 articles). Sixty-seven articles reported calibration ranges, which varied significantly for the various drugs assayed: some in the range of $0.1-0.5 \mathrm{ng} / \mathrm{mL}$ and others 1250 $200000 \mathrm{ng} / \mathrm{mL}$. Also, 61 articles reported $\mathrm{R}^{2}$ values (0.964-1.0) for almost all analytes under consideration. Sixty-three articles reported percent recoveries mostly between $61.0 \%$ to $114.0 \%$, with only two articles reporting recoveries of $4.9 \%$ and $36 \%$. Out of the 71 reviewed articles, 56 reported intermediate precision values ranging between $0.331 \%$ to $19.76 \%$, which is within the acceptable limit of $20 \%$. This review will serve as a guide for research and/or routine clinical monitoring of aminoglycosides in biological matrices.

C2022 by the authors. This article is an open-access article distributed under the terms and conditions of the Creative Commons Attribution license (http://creativecommons.org/licenses/by/4.0/).
\end{abstract}

\section{Keywords}

PK studies; Therapeutic drug monitoring; Biological matrices.

\section{Introduction}

Aminoglycosides are broad-spectrum antibiotics that are used in the treatment of gram-negative bacterial infections [1,2]. Aminoglycosides elicit their pharmacological effect by binding to the 16S rRNA ribosomal subunit of bacteria and blocking mRNA translation, altering protein synthesis. Structurally, this class of antibiotics has amino sugars in their core connected via glycosidic linkages to a dibasic aminocyclitol [3]. Aminoglycosides are relatively hydrophilic, hence, rarely undergo biotransformation in vivo. Aminoglycosides, to some extent, bind to plasma proteins and are excreted entirely unchanged in urine [4]. Streptomycin, netilmicin, tobramycin, kanamycin, spectinomycin, gentamicin, neomycin, amikacin, and 
paromomycin are examples of aminoglycosides $[5,6]$.

Aminoglycosides are known to possess nephrotoxic and ototoxic potentials, limiting their clinical use [2]. In addition, due to their narrow therapeutic index, therapeutic monitoring is required for aminoglycosides. Especially among patients with underlying renal problems, monitoring aminoglycosides in biological matrices ensures optimal therapy and reduces toxicity [7].

There are several validated bioanalytical methods used to quantitatively determine levels of aminoglycosides in biological matrices. Microbiological assays, radioimmunoassay (RIA), radioenzymatic assays, fluorescence polarization immunoassay (FPIA), high-performance liquid chromatography (HPLC), gas chromatography (GC) and mass spectrometric techniques are some common examples [8-12]. Of these methods, HPLC is the most preferred or routinely used [13]. Indeed, several studies have used HPLC for the bioanalysis of aminoglycosides [14-16]. Although there are recently published reviews on aminoglycosides, the focus of these reviews have been a) pre-treatment and analysis methods of aminoglycosides in food [17], b) challenges in the development of analytical test procedures for aminoglycosides [18], and c) determination of kanamycin by high-performance liquid chromatography [19].

The current review focuses on liquid chromatographic methods employed for the bioanalysis of aminoglycosides. A total of 20 aminoglycosides were reported by 71 articles (total number reviewed). The aminoglycosides were gentamicin [20], netilmicin [21], amikacin [22], tobramycin [23], dibekacin [24], sisomicin [25], astromicin [25], micronomicin [25], kanamycin [26], streptomycin [27], neomycin [14], isepamicin [28], geneticin [29], dihydrostreptomycin [30], paromomycin [31], apramycin [5], hygromycin [5], etimicin [32], arbekacin [33], and spectinomycin [34]. The aforementioned aminoglycosides obtained from either natural products or semi-synthetic derivatives of soil actinomycetes notably Streptomyces have suffix -mycin (examples are streptomycin, dihydrostreptomycin, kanamycin, apramycin, paromomycin, neomycin, tobramycin, spectinomycin, and hygromycin); and those obtained from other actinomycetes notably Micromonospora have the suffix -micin (examples are gentamicin, netilmicin, isepamicin, sisomicin, etimicin, geneticin, astromicin, and micronomicin). There are other exceptions, such as amikacin, arbekacin, and dibekacin. The constitute structures of these 20 aminoglycosides are presented in Figure 1 . In the review, gentamicin was the most reported aminoglycoside (24 articles). This is not surprising since gentamicin is often used clinically because of its low cost and high efficacy against gram-negative aerobes. Fifty (50) articles reported bioanalysis of at least one of the 20 aminoglycosides, whilst 21 articles reported analysis of more than one aminoglycoside. The highest number of aminoglycosides simultaneously assayed was $13[5]$.

Although the list of articles used in this review may not be exhaustive, suitable liquid chromatographic conditions used in assaying aminoglycosides in biological matrices have been identified. Performance metrics of the various liquid chromatographic assays have also been appraised. Also, highlights of current procedures, scope, characteristics, and limitations of chromatographic methods used in assaying aminoglycosides in biological matrices have been provided. Although this is not a systematic review, it will serve as a comprehensive reference for subsequent related research that may involve the assay of aminoglycosides.

\section{Methods}

This study reviewed relevant and accessible articles on liquid chromatographic assays of aminoglycosides in biological matrices from 1977 to 2020. Articles were retrieved from journals in online academic databases (PubMed, Science Direct, Scopus, and Google Scholar) and limited to only the English 
language. Keywords used during the search were aminoglycosides, assay, HPLC, plasma, serum, milk, cerebrospinal fluid, and urine. The searched terms used were "chromatographic assay", "aminoglycosides" and "biological matrix". Articles were excluded if they were not pertinent.

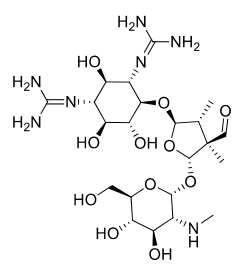

Streptomycin

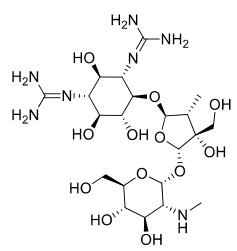

Dihydrostreptomycin

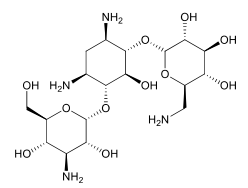

Kanamycin
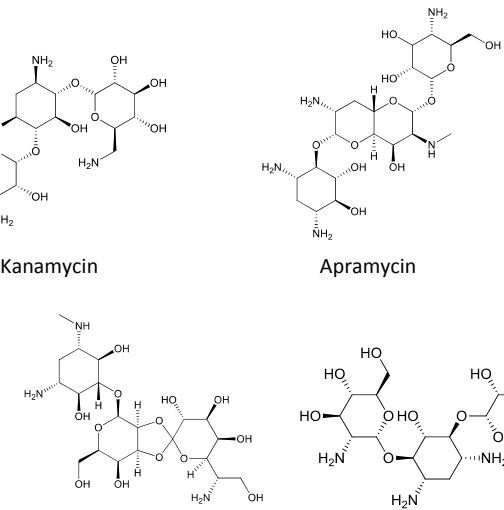

Hygromycin

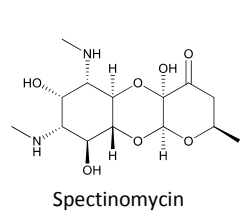

Tobramycin

Spectinomycin

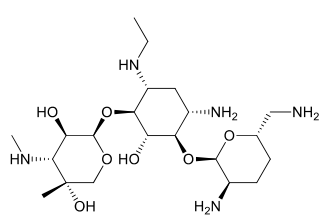

Dibekacin

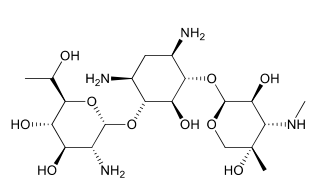

Geneticin
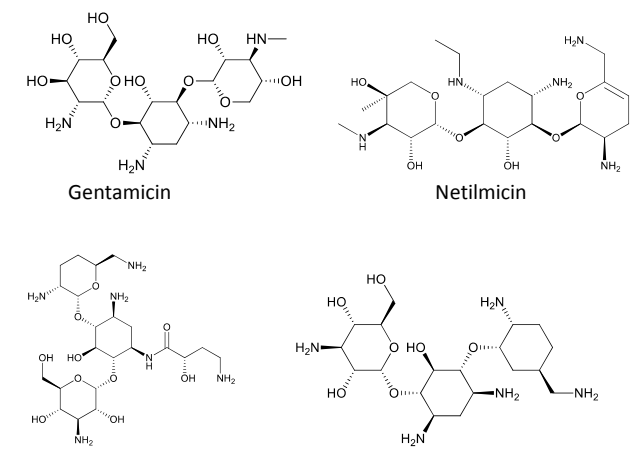

Arbekacin

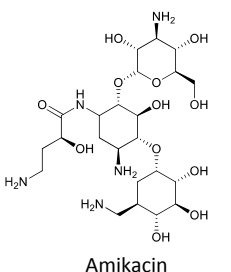

Isepamicin
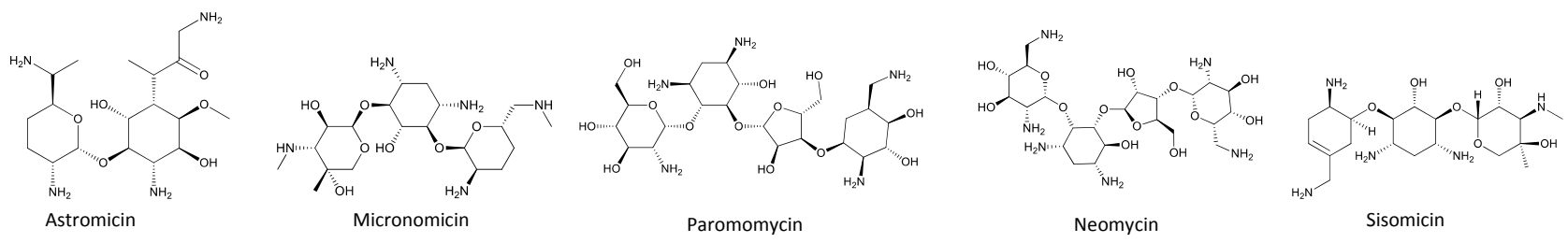

Paromomycin

Neomycin

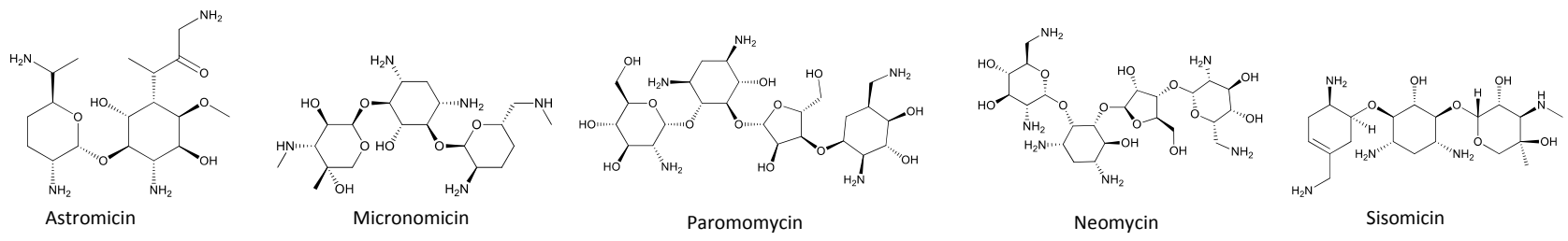

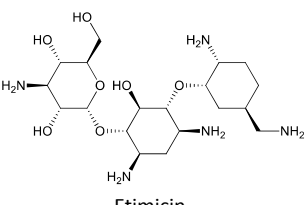

Etimicin

Figure 1. Structures of the 20 aminoglycosides reported by the various articles reviewed

\section{Aminoglycosides}

Sixty-seven (67) out of the 71 articles reported the use of HPLC in the bioanalysis of aminoglycosides, while four articles used ultra-performance liquid chromatography (UPLC) [35]. The relevant aspect of liquid chromatographic conditions used in the various articles, such as matrix, sample preparation, flow rate, column selection, mobile phase, and detection, have been summarized (Table 1). 
Table 1. HPLC conditions for bioanalysis of aminoglycosides.

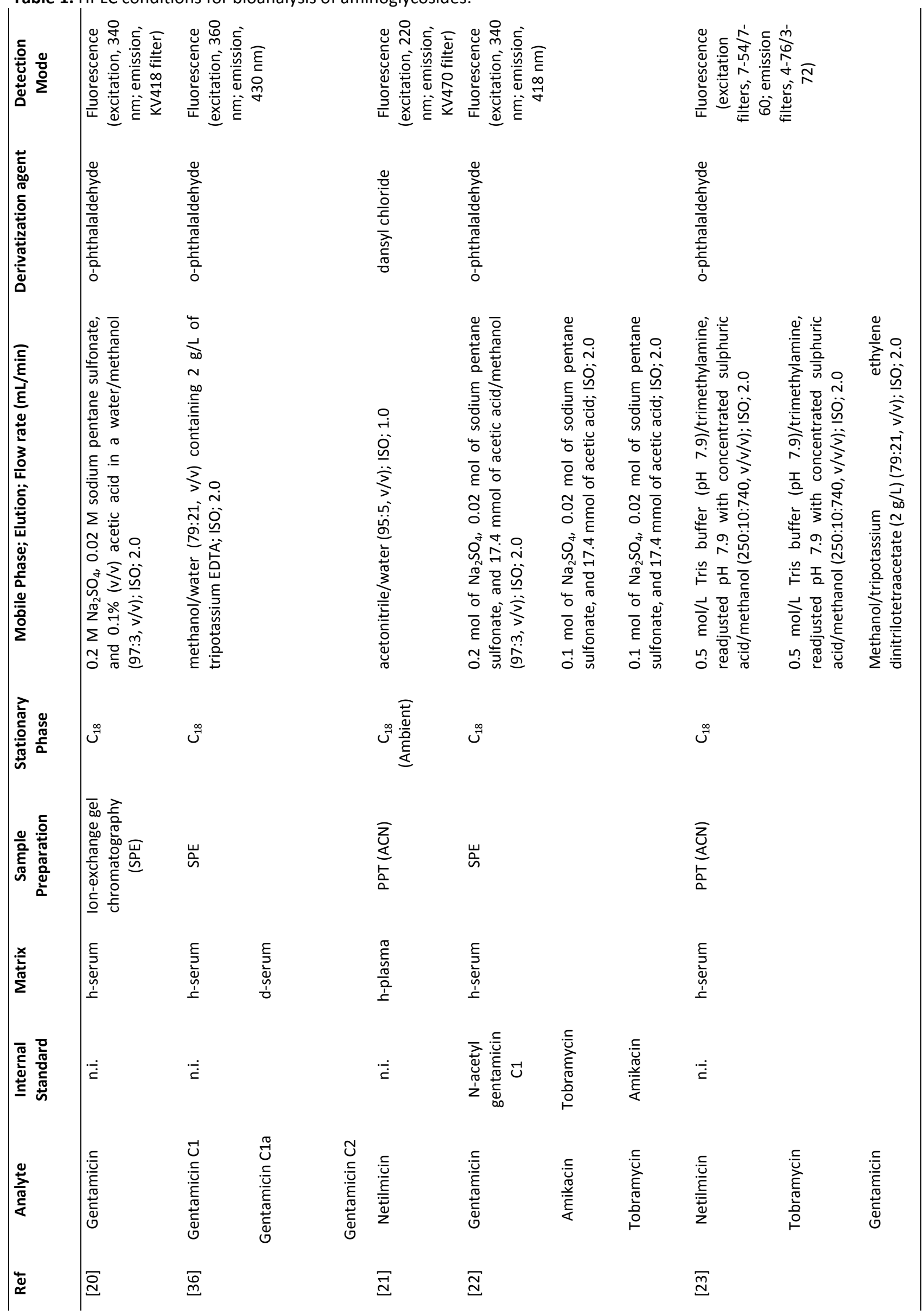


Table 1. Contined...

\begin{tabular}{|c|c|c|c|c|c|c|c|c|}
\hline 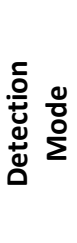 & 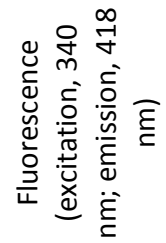 & 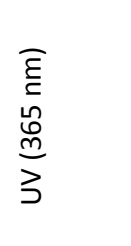 & 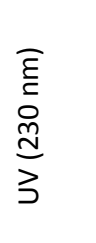 & 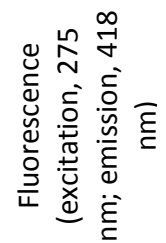 & 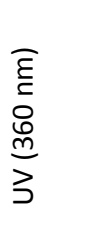 & 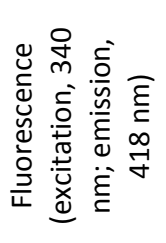 & 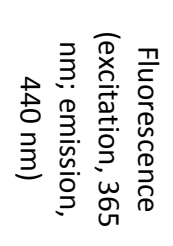 & 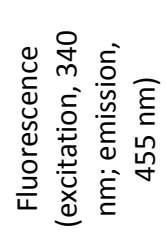 \\
\hline 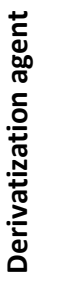 & $\begin{array}{l}\frac{0}{0} \\
\frac{1}{2} \\
\frac{0}{0} \\
\frac{0}{0} \\
\frac{0}{0} \\
\frac{1}{1} \\
\frac{0}{1} \\
0\end{array}$ & 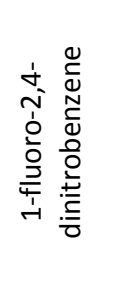 & 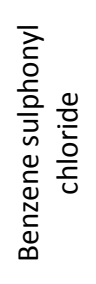 & 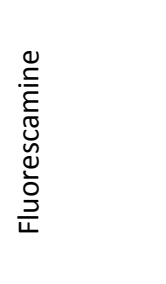 & 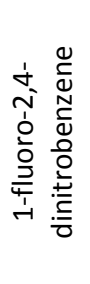 & 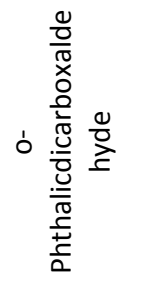 & $\begin{array}{l}\frac{0}{0} \\
\frac{1}{2} \\
\frac{0}{0} \\
\frac{0}{0} \\
\frac{0}{0} \\
\frac{1}{2} \\
\frac{0}{1} \\
0\end{array}$ & $\begin{array}{l}\frac{0}{0} \\
\frac{1}{2} \\
\frac{0}{0} \\
\frac{0}{0} \\
\frac{\pi}{0} \\
\frac{1}{1} \\
\frac{1}{0} \\
\frac{1}{0}\end{array}$ \\
\hline 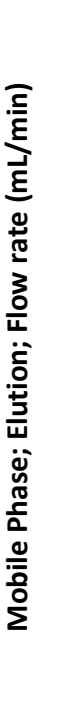 & 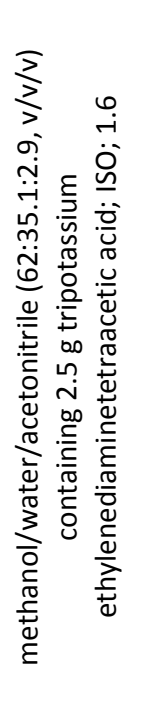 & 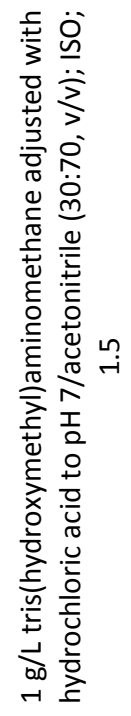 & 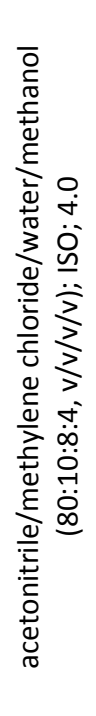 & 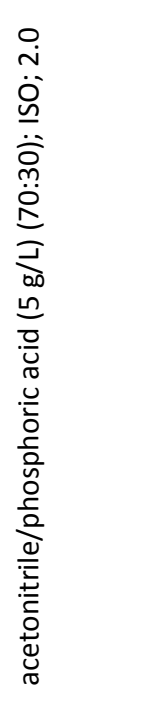 & 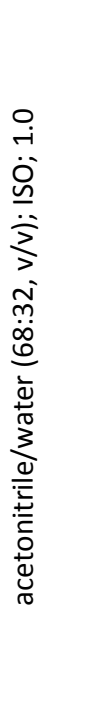 & 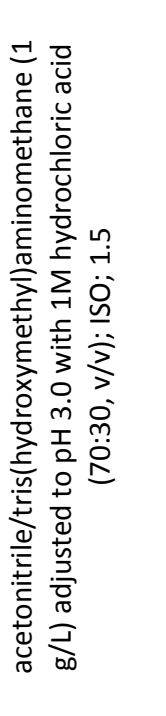 & 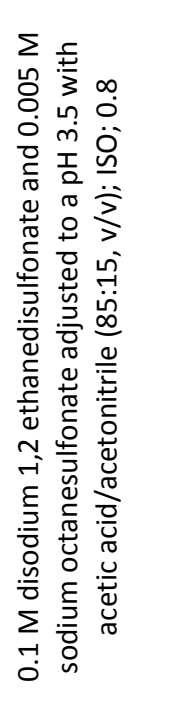 & 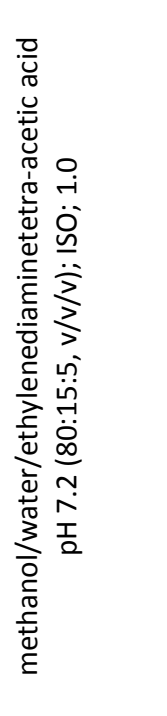 \\
\hline 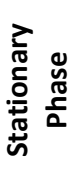 & 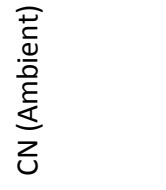 & 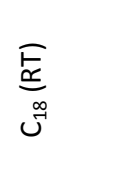 & $\stackrel{\sim}{~}^{\infty}$ & 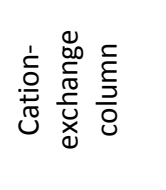 & 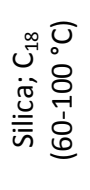 & 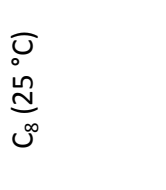 & 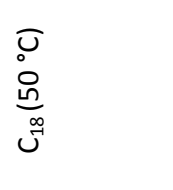 & 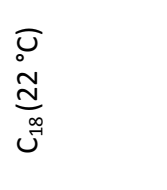 \\
\hline 总 & $\begin{array}{l}\bar{z} \\
\frac{u}{\leq} \\
\frac{5}{a}\end{array}$ & 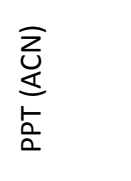 & 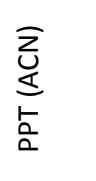 & 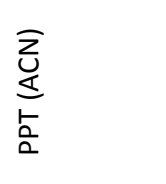 & 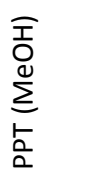 & 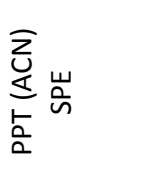 & 占 & 㫕 \\
\hline 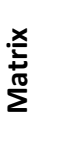 & 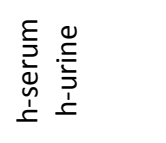 & 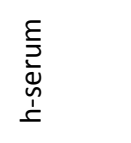 & 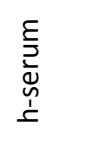 & 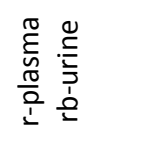 & 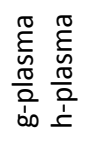 & 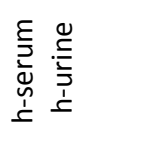 & 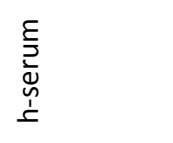 & 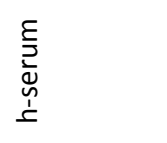 \\
\hline 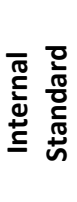 & 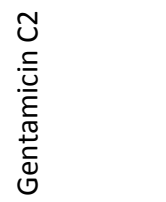 & $\dot{\dot{E}}$ & 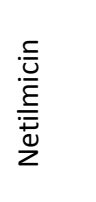 & $\dot{\dot{x}}$ & $\dot{\dot{E}}$ & 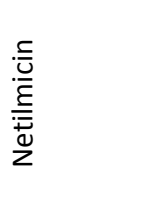 & $\dot{\dot{x}}$ & 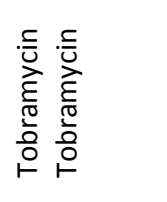 \\
\hline 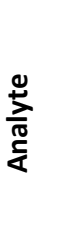 & $\begin{array}{l}\frac{5}{0} \\
\text { है } \\
\frac{0}{0} \\
\frac{0}{0} \\
\qquad\end{array}$ & 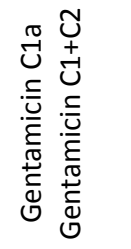 & 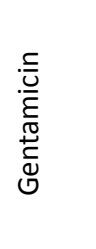 & 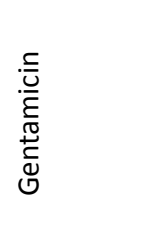 & 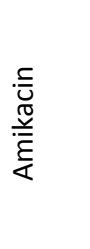 & 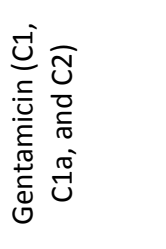 & 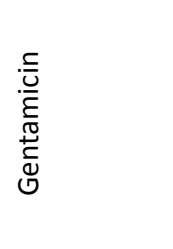 & 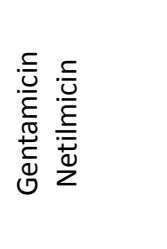 \\
\hline 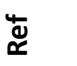 & $\underset{\tilde{m}}{\bar{m}}$ & $\stackrel{\infty}{\stackrel{\infty}{=}}$ & శ్ & 守 & $\underset{\Xi}{\vec{d}}$ & $\underset{\mathcal{I}}{\check{Z}}$ & $\underline{\bar{g}}$ & 导 \\
\hline
\end{tabular}


Table 1. Contined...

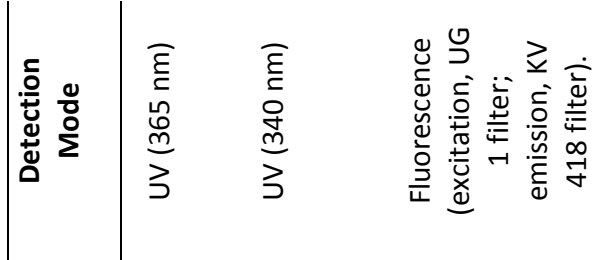

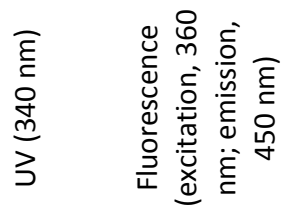

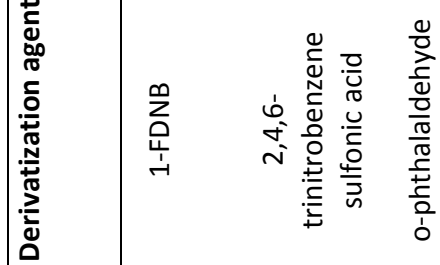

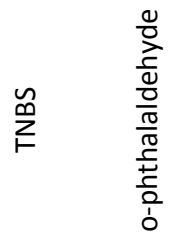

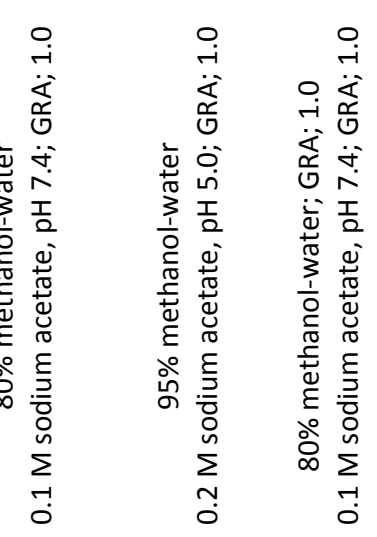

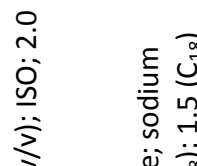
年

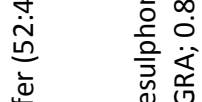

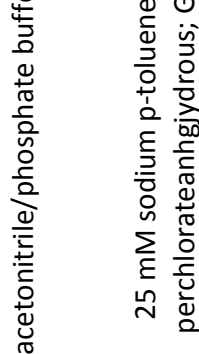

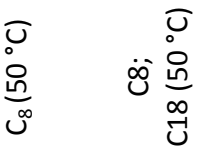
岕
岕
言

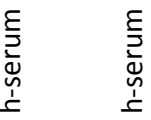

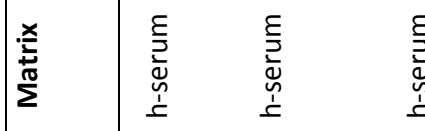

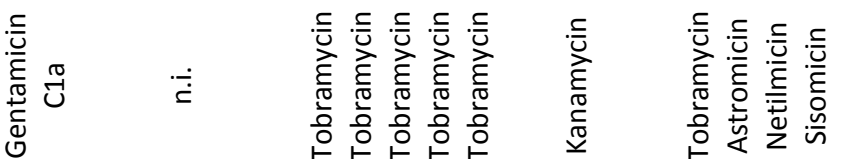

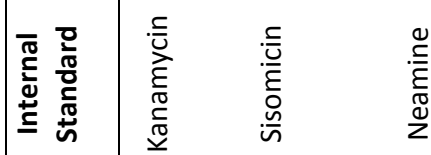
旁

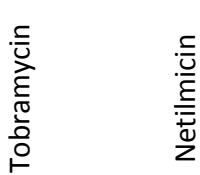

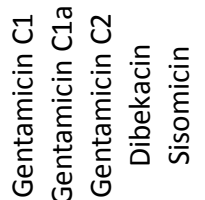

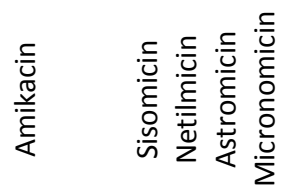
离 孪 急 导
$\sqrt[\bar{g}]{\sqrt{d}}$ 
Table 1. Contined...

\begin{tabular}{|c|c|c|c|c|c|c|c|c|}
\hline 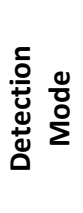 & 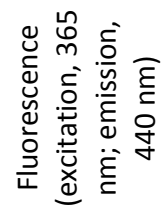 & 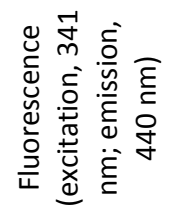 & & 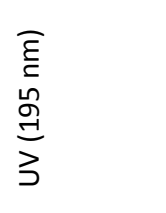 & 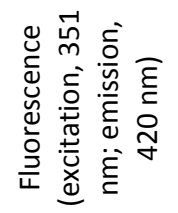 & 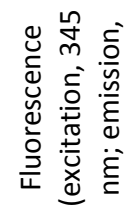 & $\begin{array}{l}\xi \\
\stackrel{\varepsilon}{\Xi} \\
m \\
\stackrel{\sim}{q}\end{array}$ & 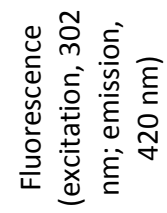 \\
\hline 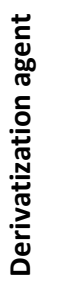 & $\begin{array}{l}\frac{0}{0} \\
\frac{1}{2} \\
\frac{0}{0} \\
\frac{0}{0} \\
\frac{\pi}{0} \\
\frac{1}{1} \\
\frac{1}{2} \\
\frac{0}{1} \\
0\end{array}$ & $\begin{array}{l}\frac{0}{0} \\
\frac{\lambda}{0} \\
\frac{0}{0} \\
\frac{0}{0} \\
\frac{0}{0} \\
\frac{5}{c} \\
\frac{0}{1} \\
0\end{array}$ & & & 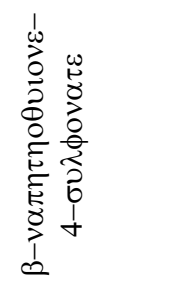 & $\begin{array}{l}\frac{0}{0} \\
\frac{1}{2} \\
\frac{0}{0} \\
\frac{0}{0} \\
\frac{0}{0} \\
\frac{1}{1} \\
\frac{1}{0} \\
0 \\
0\end{array}$ & & 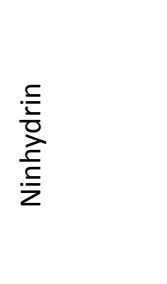 \\
\hline 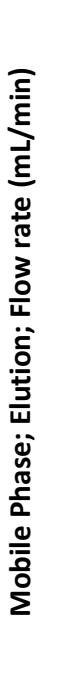 & 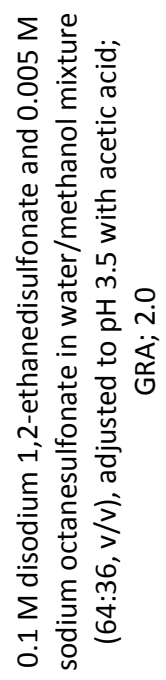 & 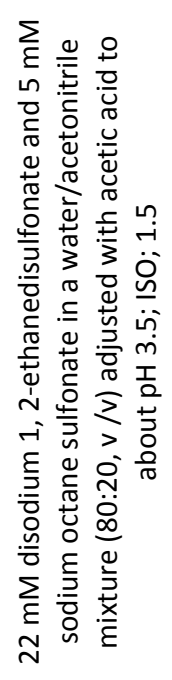 & 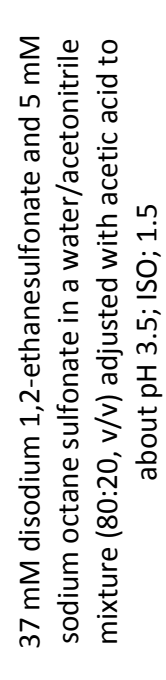 & 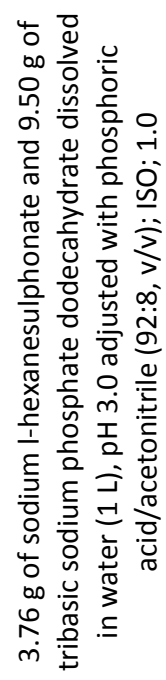 & 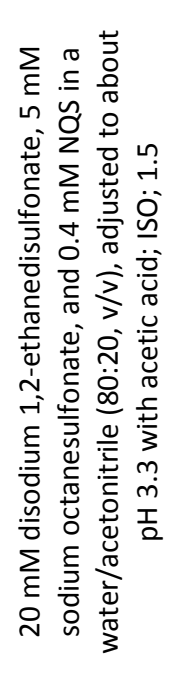 & 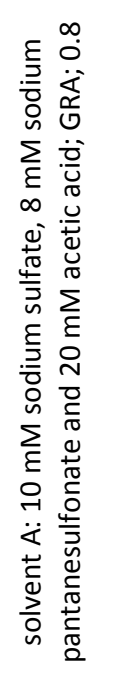 & 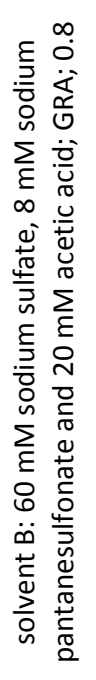 & 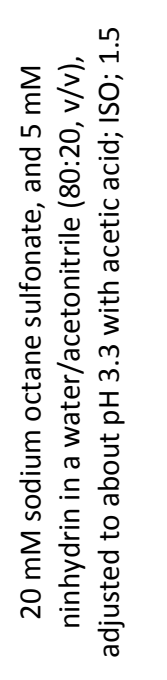 \\
\hline 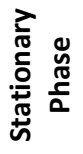 & $\stackrel{\infty}{\cup}^{-}$ & $\stackrel{\infty}{\cup}^{-1}$ & & 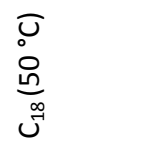 & 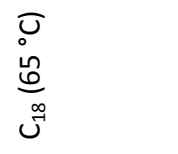 & 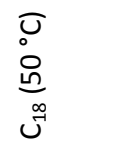 & & 䞌 \\
\hline$\frac{0}{\frac{0}{0}}$ & 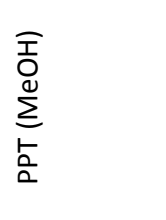 & 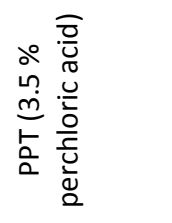 & & 崩 & 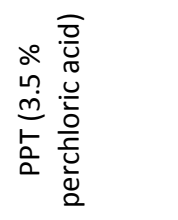 & 崩 & & 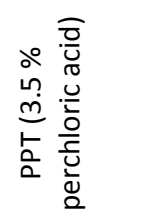 \\
\hline 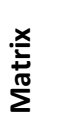 & 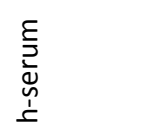 & 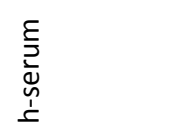 & & 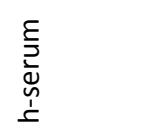 & 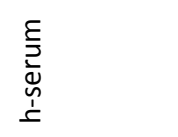 & 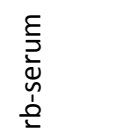 & & 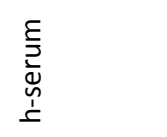 \\
\hline 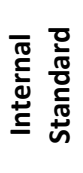 & 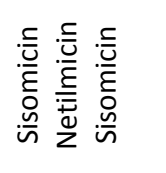 & $\dot{\check{c}}$ & & 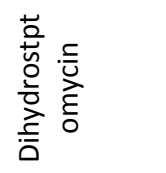 & $\dot{\dot{\Phi}}$ & $\dot{\dot{I}}$ & & $\stackrel{\dot{\Phi}}{\dot{\Sigma}}$ \\
\hline$\frac{\stackrel{0}{\frac{1}{2}}}{\frac{\pi}{\pi}}$ & 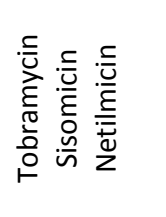 & 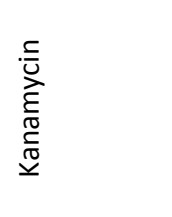 & 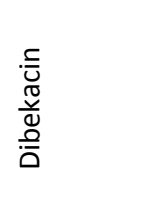 & 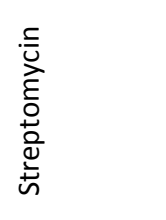 & 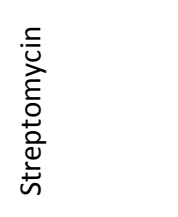 & 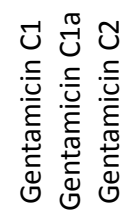 & & 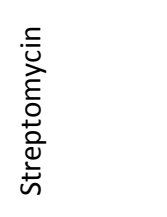 \\
\hline 芯 & $\stackrel{\infty}{+}$ & 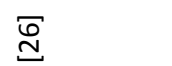 & & $\underset{\beth}{\Xi}$ & 宁 & 뭄 & & ت્ \\
\hline
\end{tabular}


Table 1. Contined...

\begin{tabular}{|c|c|c|c|c|c|c|c|c|c|}
\hline 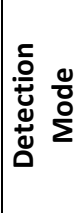 & 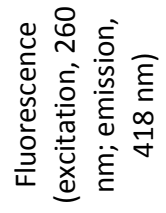 & 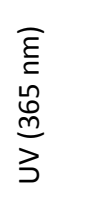 & 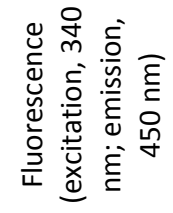 & 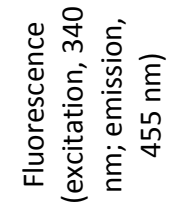 & 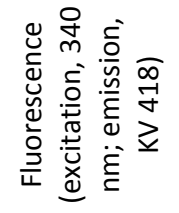 & 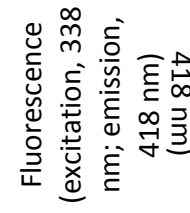 & 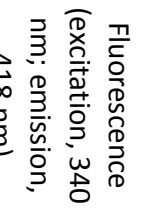 & $\begin{array}{l}\bar{\xi} \\
\vdots \\
0 \\
⿱ 亠 䒑 \\
m \\
\vdots\end{array}$ & 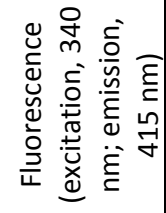 \\
\hline 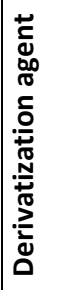 & $\begin{array}{l}\frac{0}{0} \\
\text { ते } \\
\frac{0}{0} \\
\frac{0}{0} \\
\frac{\pi}{0} \\
\frac{1}{1} \\
\frac{1}{0} \\
\frac{1}{1} \\
0\end{array}$ & $\begin{array}{l}\frac{0}{0} \\
\frac{1}{2} \\
\frac{0}{0} \\
\frac{0}{0} \\
\frac{10}{0} \\
\frac{c}{1} \\
\frac{1}{0} \\
0\end{array}$ & $\begin{array}{l}\frac{0}{0} \\
\frac{1}{d} \\
\frac{1}{0} \\
\frac{0}{0} \\
\frac{0}{0} \\
\frac{1}{1} \\
\frac{1}{2} \\
\frac{0}{0}\end{array}$ & & $\begin{array}{l}\frac{0}{0} \\
\frac{1}{c} \\
\frac{0}{0} \\
\frac{0}{0} \\
\frac{0}{0} \\
\frac{1}{4} \\
\frac{1}{0} \\
\frac{0}{1}\end{array}$ & $\begin{array}{l}\frac{0}{0} \\
\frac{1}{d} \\
\frac{0}{0} \\
\frac{0}{0} \\
\frac{0}{0} \\
\frac{1}{t} \\
\frac{1}{0} \\
0\end{array}$ & $\begin{array}{l}\frac{0}{0} \\
\frac{1}{d} \\
\frac{0}{0} \\
\frac{0}{0} \\
\frac{\pi}{\pi} \\
\frac{1}{1} \\
\frac{1}{0} \\
\frac{0}{0} \\
0\end{array}$ & 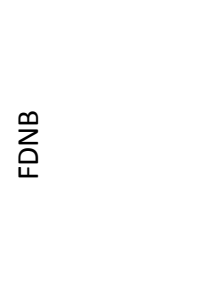 & $\begin{array}{l}\frac{0}{0} \\
\frac{1}{c} \\
\frac{0}{0} \\
\frac{0}{0} \\
\frac{0}{0} \\
\frac{1}{1} \\
\frac{1}{0} \\
\frac{0}{1}\end{array}$ \\
\hline 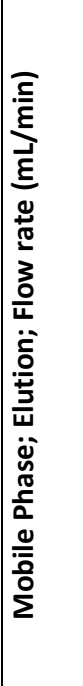 & 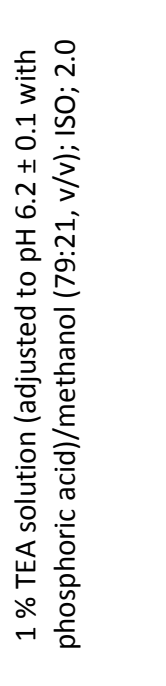 & 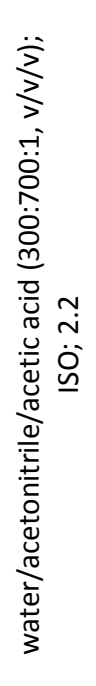 & 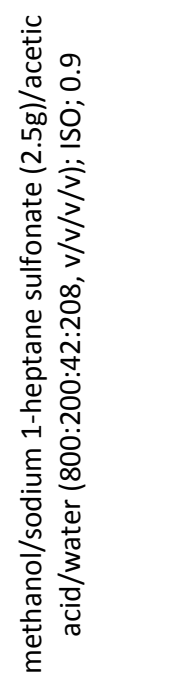 & 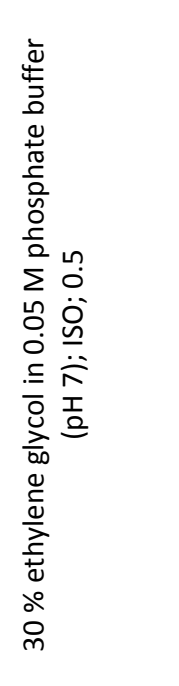 & 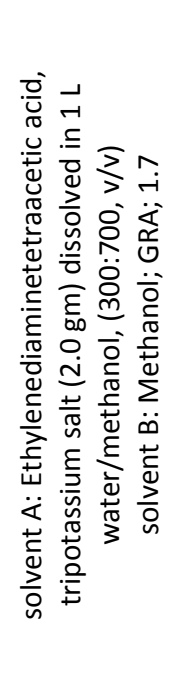 & 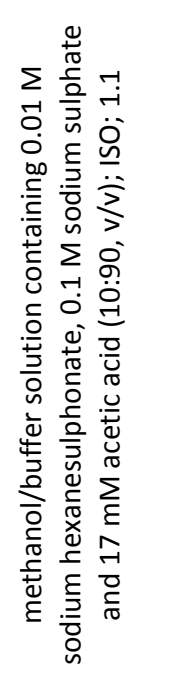 & 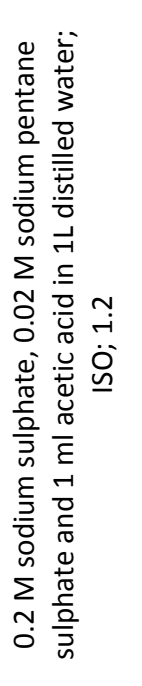 & 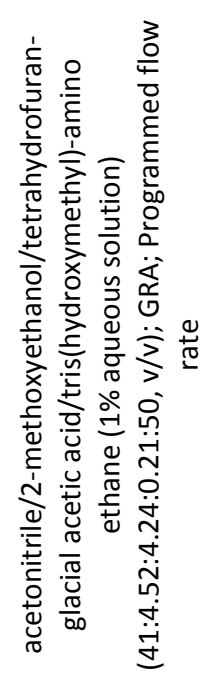 & 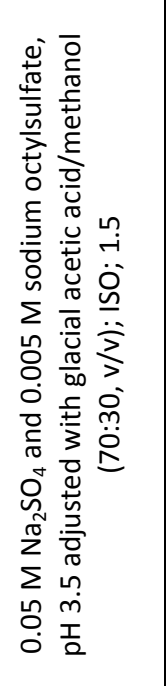 \\
\hline 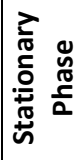 & $\stackrel{\sim}{\sim}^{\prime}$ & 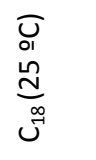 & $\cup^{\infty}$ & $\cup^{\infty}$ & 呈 & 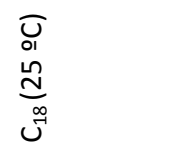 & $\stackrel{\sim}{~}^{\infty}$ & 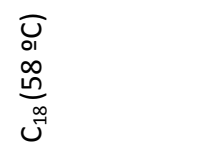 & 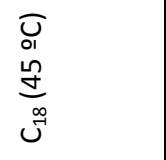 \\
\hline 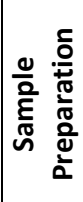 & ü & 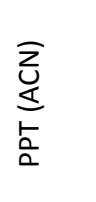 & 峁 & 剻 & 峁 & ü & 幽 & 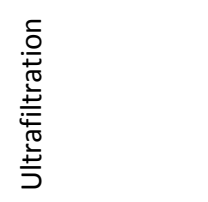 & 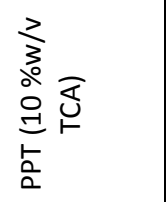 \\
\hline 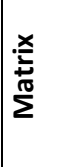 & 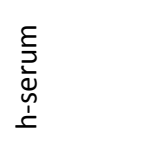 & 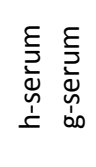 & $\begin{array}{l}\text { ص̃ } \\
\text { Lิ }\end{array}$ & $\begin{array}{l}\varepsilon \\
\frac{\xi}{2} \\
\bar{\nu} \\
\frac{0}{2}\end{array}$ & & 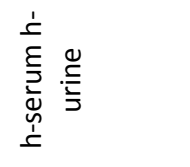 & 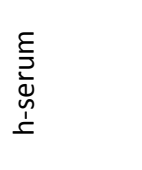 & 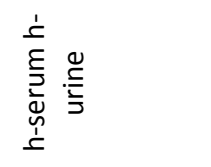 & $\begin{array}{l}\frac{\pi}{6} \\
\frac{\tilde{n}}{0} \\
\frac{0}{0} \\
\frac{0}{1}\end{array}$ \\
\hline 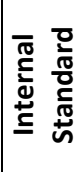 & $\dot{\bar{C}}$ & 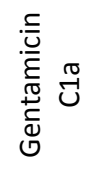 & $\dot{\dot{C}}$ & $\dot{\dot{C}}$ & $\dot{\dot{C}}$ & 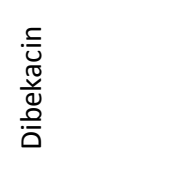 & 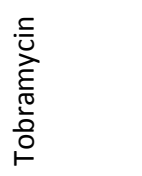 & $\dot{\dot{\check{C}}}$ & $\dot{\dot{\Sigma}}$ \\
\hline 䇋 & 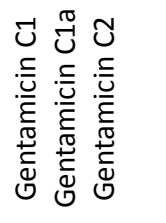 & 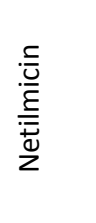 & 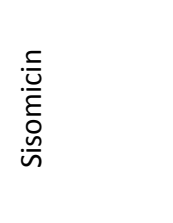 & 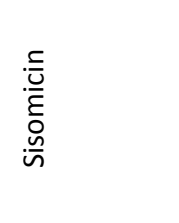 & $\begin{array}{l}\frac{c}{0} \\
\stackrel{\vec{c}}{0} \\
\frac{d}{z}\end{array}$ & 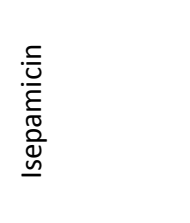 & 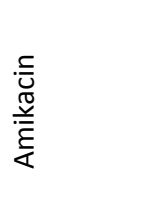 & 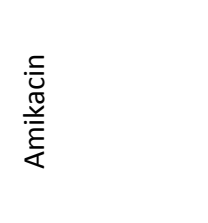 & 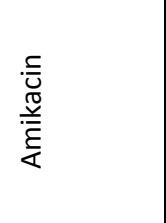 \\
\hline $\begin{array}{l}\stackrel{4}{\triangle} \\
\propto\end{array}$ & $\underset{\widetilde{n}}{\widetilde{n}}$ & 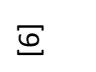 & 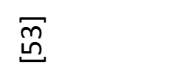 & 它 & 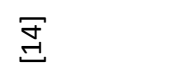 & $\stackrel{\infty}{\sim}$ & 朚 & 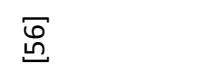 & $\sqrt{\text { 公 }}$ \\
\hline
\end{tabular}


Table 1. Contined...

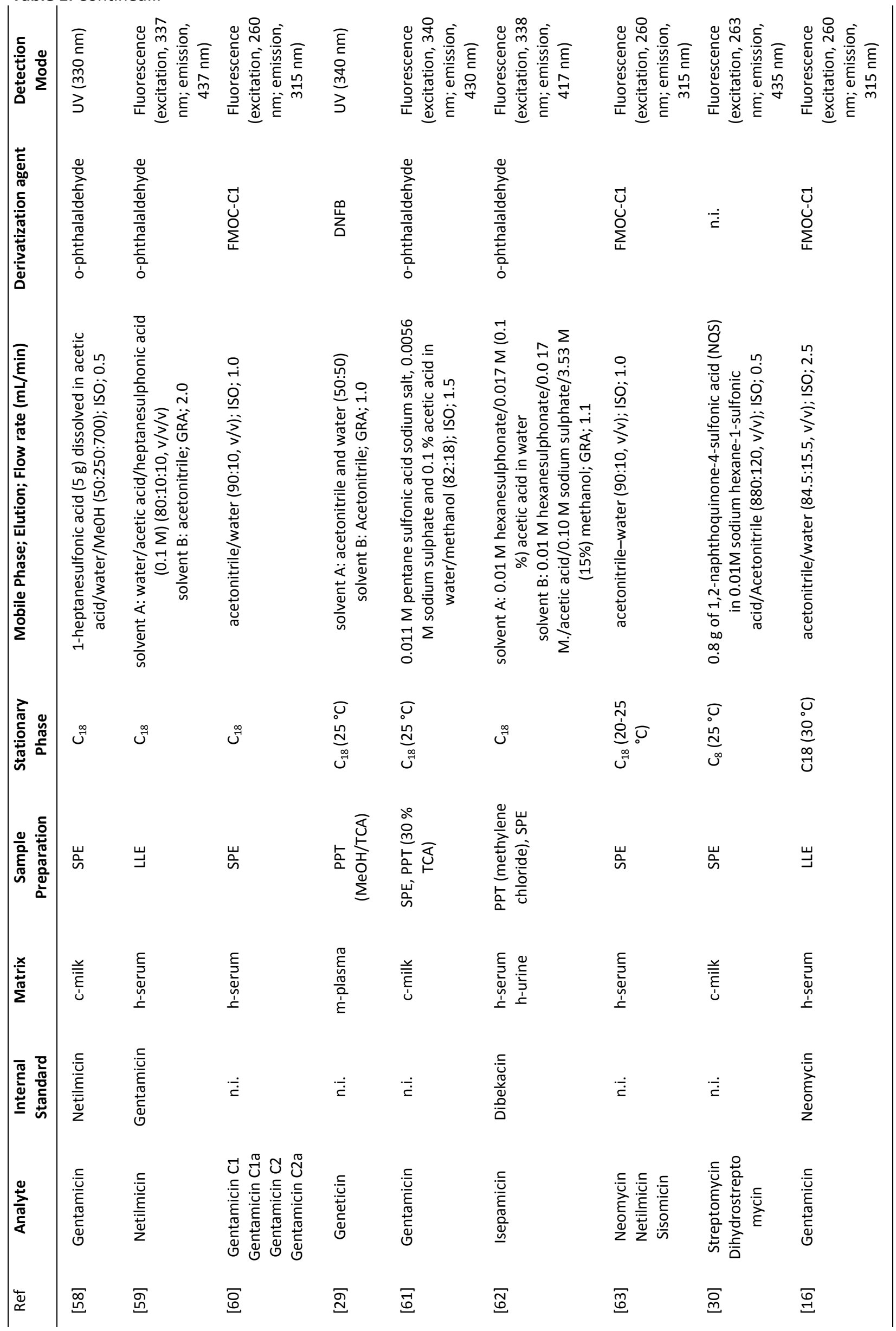


Table 1. Contined...

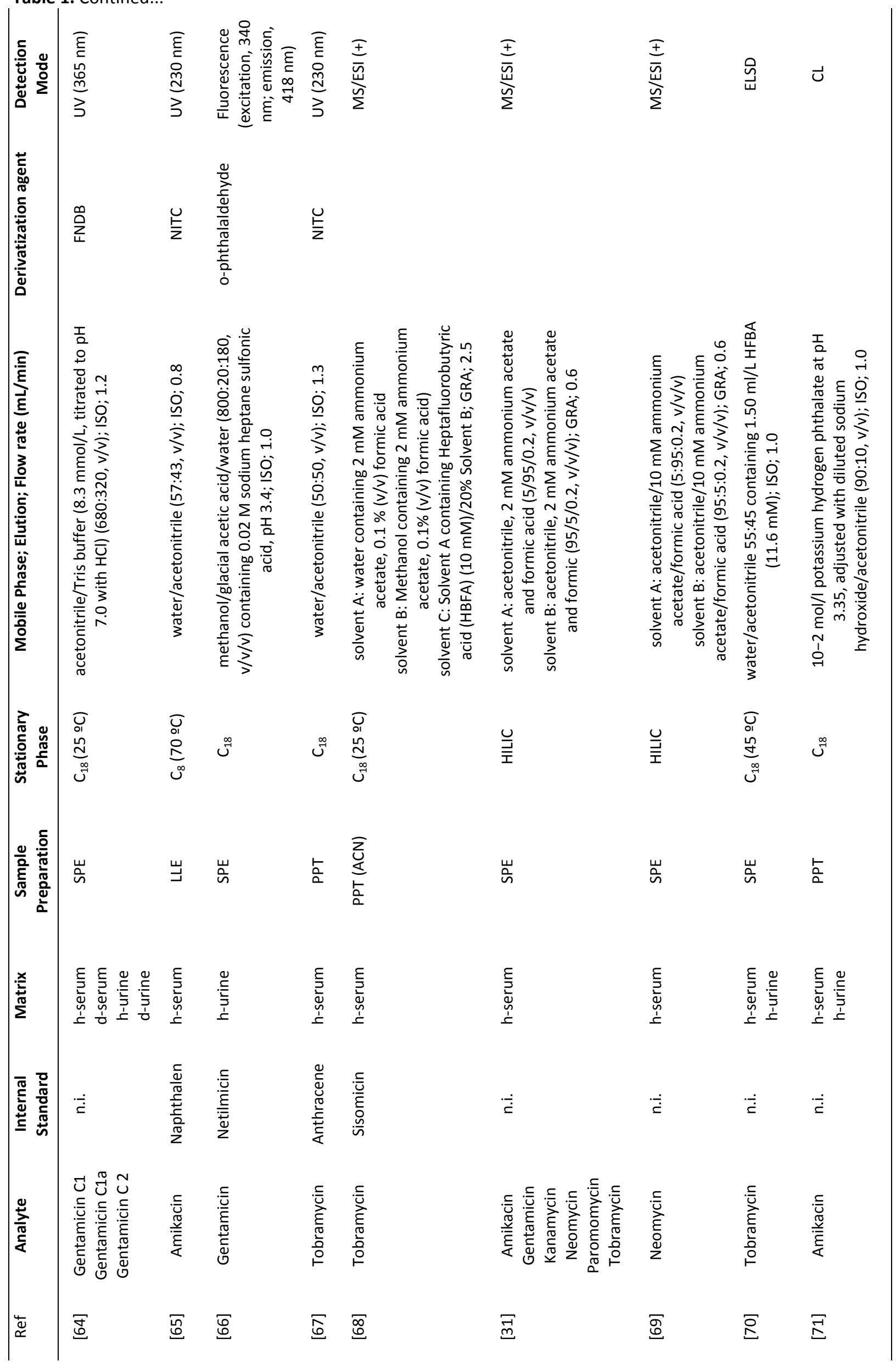


Table 1. Contined...

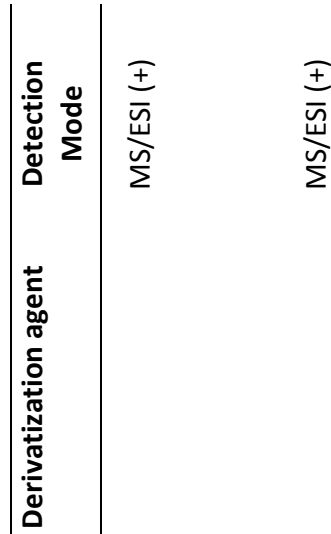

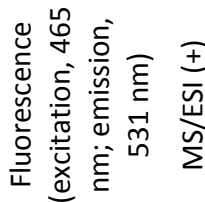

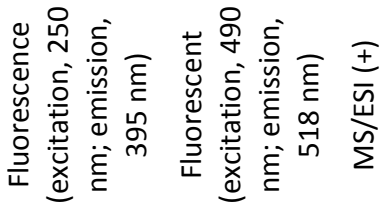

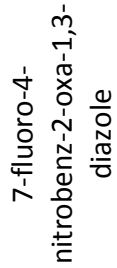

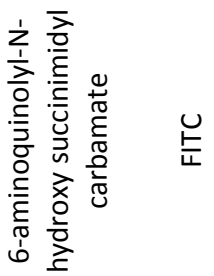

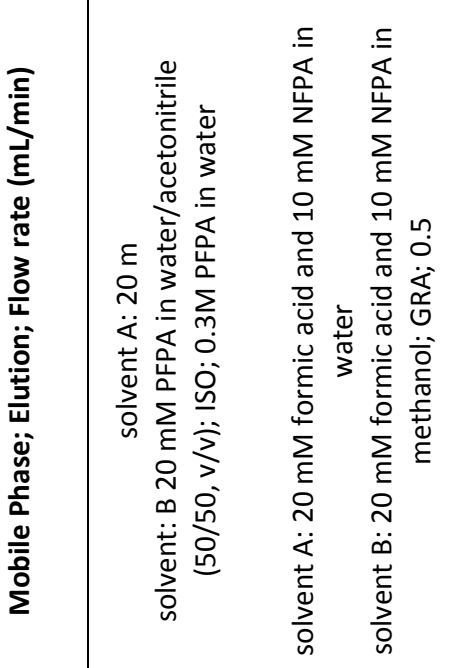

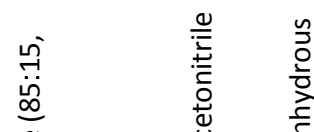
管 究员

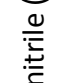

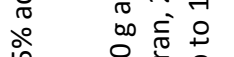

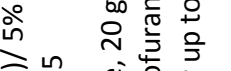

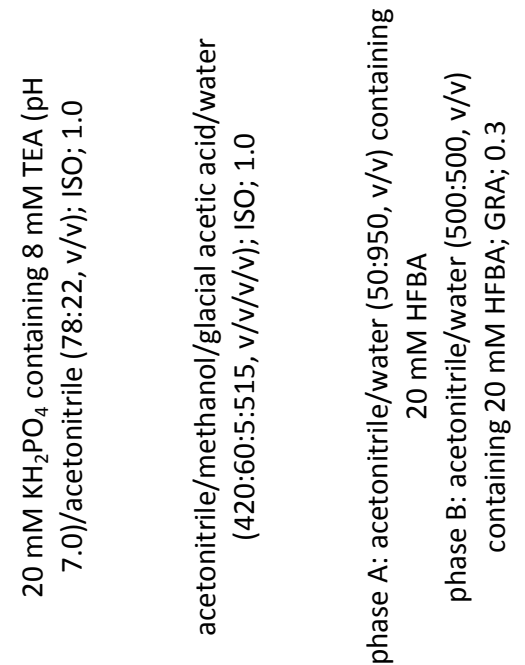

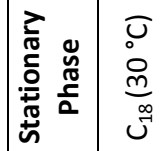

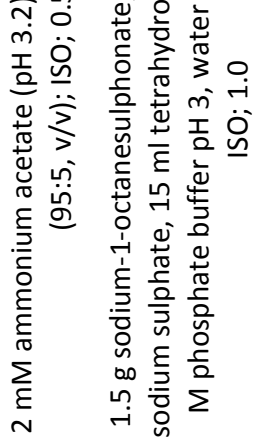
离
m

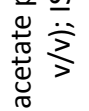

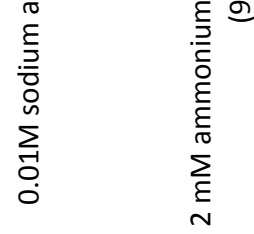
$\begin{array}{ll}0 & 0 \\ 0 & 0 \\ 0 & 0 \\ 0 & 0 \\ j & j\end{array}$

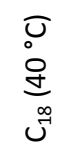

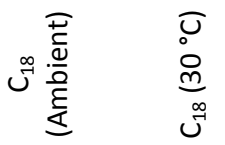

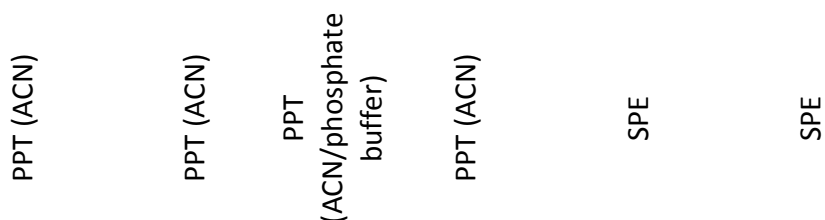

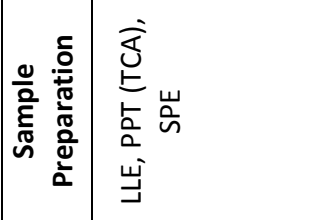
究

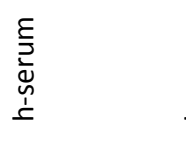

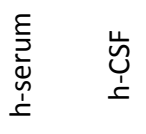

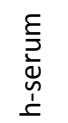

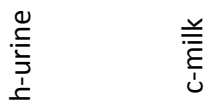
$\dot{\bar{\varepsilon}}$

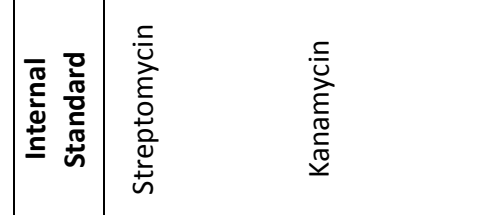

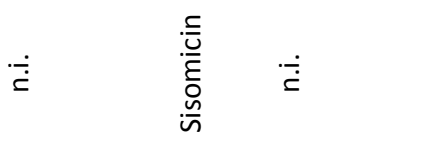

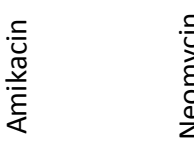

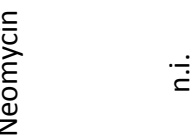

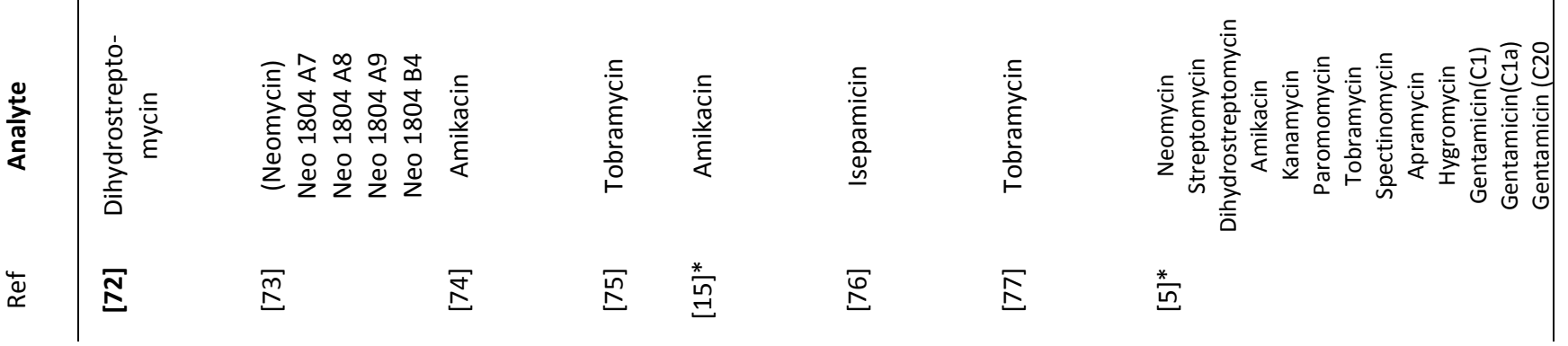


Table 1. Contined...

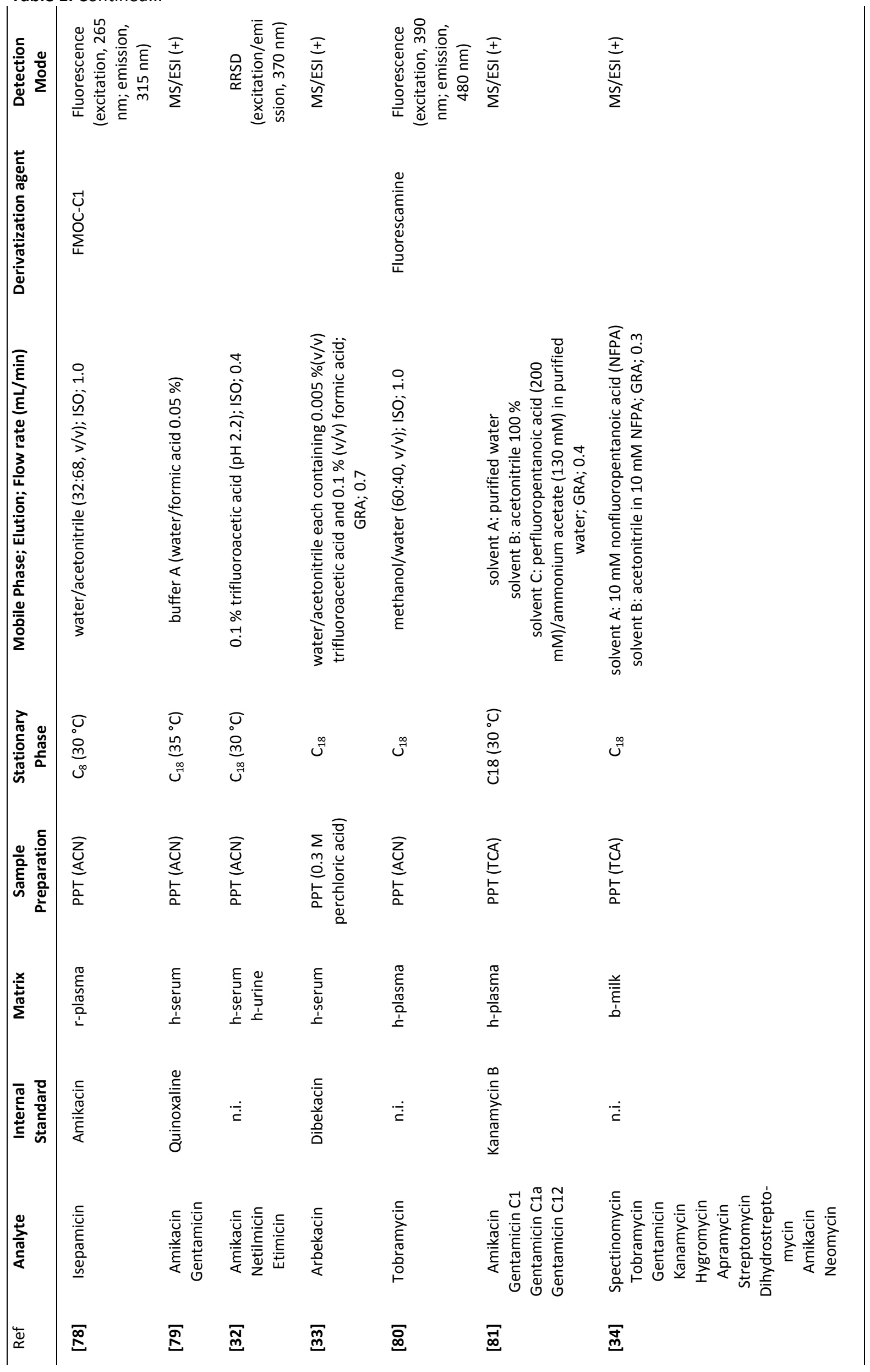


Table 1. Contined...

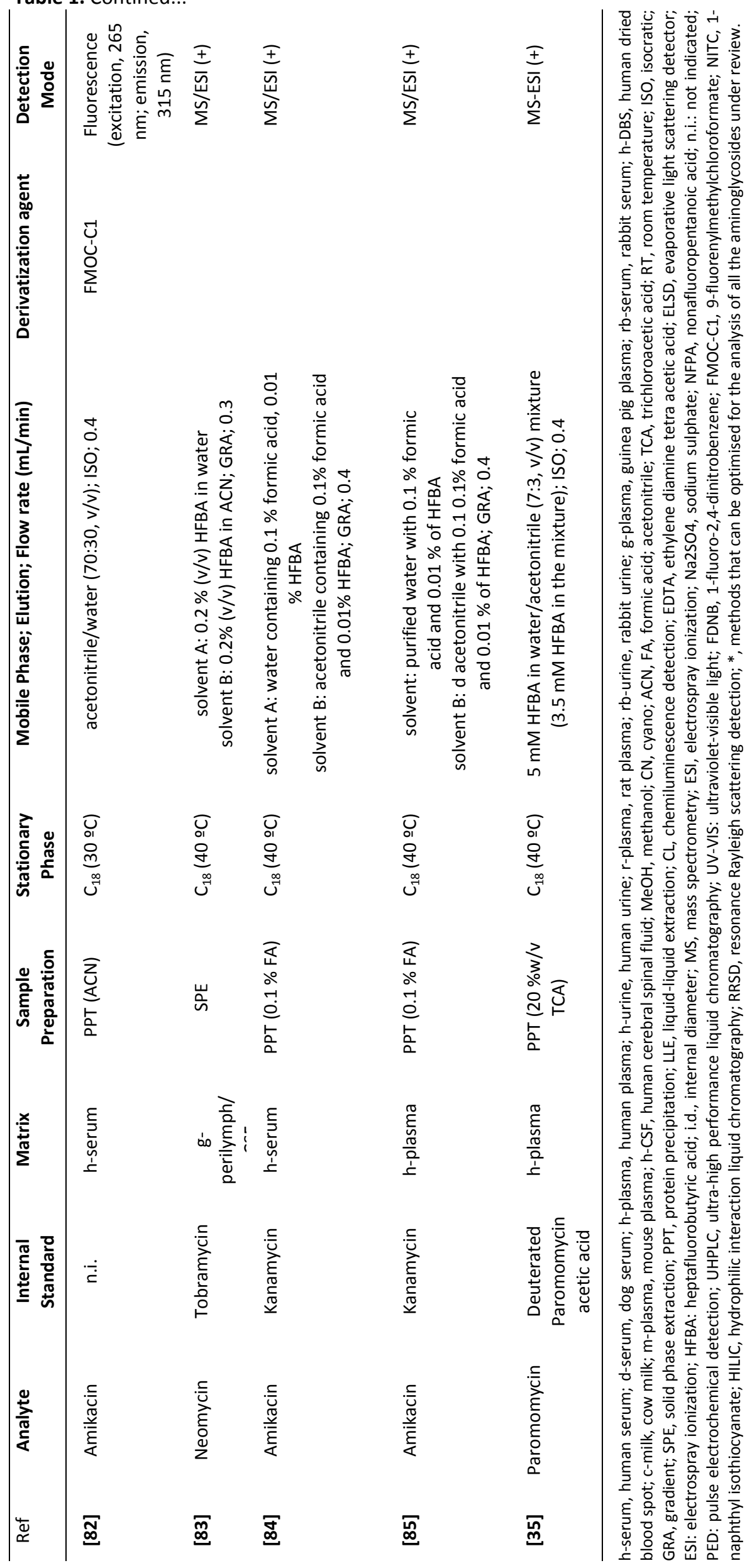




\section{Sample cleanup}

The matrix in which a drug is found can affect bioanalysis. Also, matrices can compromise the sensitivity and selectivity of bioanalysis methods [86-89], which is deemed a "matrix effect". The matrix effect could be due to endogenous or exogenous agents. Some endogenous substances include salts, carbohydrates, amines, urea, lipids, peptides, and metabolites [90,91]. Exogenous substances may include anticoagulants, blood preservatives, and mobile-phase additives such as buffer salts [92,93]. Sample cleanup is required to isolate analyte(s) of interest from a matrix.

Sample cleanup tends to reduce or remove matrix components and concentrate the analyte(s). This process improves assay sensitivity and selectivity. An optimal sample cleanup system should be capable of minimizing matrix effect while ensuring reliable extraction recovery [94]. Basically, there are three sample preparation methods that can be applied during HPLC bioanalysis of aminoglycosides; protein precipitation (PPT), solid-phase extraction (SPE), and liquid-liquid extraction (LLE) [4]. All the articles reviewed provided sample preparation methods; 41 used PPT, 33 SPE, and 4 LLE. One article reported the use of ultrafiltration as a sample preparation method [56], 6 articles reported a combination of PPT and SPE [25,42,46,47,61,62], and 1 article used a combination of PPT, SPE, and LLE [72].

Solvents that were used for PPT included acetonitrile for the extraction of netilmicin, amikacin, tobramycin, gentamicin, etimicin, neomycin, and isepamicin $[6,15,21,23,32,37-40,42,68,73-76,78,79,82,86]$; trichloroacetic acid for the extraction of geneticin, spectinomycin, tobramycin, gentamicin, kanamycin, hygromycin, apramycin, streptomycin, dihydrostreptomycin, amikacin, neomycin, and paromomycin $[29,34,35,57,61,72,81]$; methanol for the extraction of geneticin, amikacin, tobramycin, sisomicin, and netilmicin [29,41,48]; perchloric acid for the extraction of kanamycin, dibekacin, arbekacin, and streptomycin [26,33,49,51]; formic acid for the extraction of amikacin [84,85]; and methylene chloride for the extraction of isepamicin [62]. PPT aids in reducing interference during derivatization [7], and this may have accounted for its use by 41 articles.

SPE is useful in isolating polar analytes such as aminoglycosides. SPE has also been proven to be useful when the volume of a matrix is high [4]. Preparation of matrices containing aminoglycosides using SPE tends to give well reproducible recovery [95]. Despite these merits, SPE is relatively expensive compared to PPT. In this review, 33 of the articles reported the use of SPE as a sample preparation technique for the extraction of gentamicin, amikacin, tobramycin, sisomicin, netilmicin, astromicin, micronomicin, streptomycin, neomycin, isepamicin, dihydrostreptomycin, kanamycin, paromomycin, spectinomycin, apramycin, and hygromycin in biological matrices.

Also, four articles adopted the LLE as a sample preparation technique for the extraction of netilmicin, gentamicin, amikacin, and dihydrostreptomycin. Aminoglycosides are hydrophilic in nature, and LLE may not be ideal for extraction.

\section{Chromatographic conditions}

All articles reviewed reported chromatographic conditions, and these are highlighted in this review.

\section{Mode of chromatography}

The most-reported mode of chromatography used for bioanalysis of aminoglycosides was reversedphase HPLC (66 articles). This may be due to the polarity of the analytes of interest [96]. Other modes of chromatography for the separation of aminoglycosides used were hydrophilic interaction liquid chromatography ( 2 articles) for the analysis of amikacin, gentamicin, kanamycin, neomycin, paromomycin, 
and tobramycin [31,69]; hydrophobic interaction chromatography (1 article) for the analysis of neomycin [14]; and ion-exchange chromatography ( 1 article) for the analysis of gentamicin [40]. One article reported the use of mixed-mode chromatography using both reversed-phase and normal chromatography for the analysis of amikacin [41].

\section{Stationary phase}

The selection of a chromatographic mode of separation is dependent on the choice of the column. In this review, 63 articles reported the use of $\mathrm{C}_{18}$ columns for the separation of any of the twenty aminoglycosides; while 3 other articles used $C_{8}$ columns for the separation of streptomycin, dihydrostreptomycin, and amikacin $[30,47,65]$. Also, ion exchange for the separation of gentamicin [40]; cyano for the separation of tobramycin [37]; shielded hydrophobic phase for the separation of neomycin [14]; silica for the separation of amikacin [41]; and hydrophilic interaction columns for the separation of amikacin, gentamicin, kanamycin, neomycin, paromomycin, and tobramycin [31,69] have been reported. This implies that $\mathrm{C}_{18}$ columns may be the most appropriate for liquid chromatographic bioanalysis of aminoglycosides. In this review, columns were kept at temperatures ranging from $22^{\circ} \mathrm{C}$ to as high as $100^{\circ} \mathrm{C}$ [41]. Overall, the most widely used column temperature was $25{ }^{\circ} \mathrm{C}$ for the separation of netilmicin, amikacin, tobramycin, gentamicin, dibekacin, sisomicin, isepamicin, geneticin, streptomycin, dihydrostreptomycin, and neomycin $[6,24,28-30,42,61,64,68,73]$; followed by $30{ }^{\circ} \mathrm{C}$ for the separation of neomycin, streptomycin, dihydrostreptomycin, amikacin, kanamycin, paromomycin, tobramycin, spectinomycin, apramycin, hygromycin, gentamicin, netilmicin, etimicin, and isepamicin $[5,16,32,72,78,81,82]$; and $50{ }^{\circ} \mathrm{C}$ for the separation of sisomicin, netilmicin, astromicin, micronomicin, streptomycin, gentamicin, tobramycin, and amikacin $[25,27,43,46,47,50]$.

\section{Internal standard}

Internal standards are normally employed to offset injection volume errors and/or losses during sample extraction [97]. Forty-four (44) articles used internal standards, which include $\mathrm{N}$-acetyl gentamicin $\mathrm{C1}$ [22], tobramycin [22,24,25,44,55,83], amikacin [22,76,78], gentamicin C2 [37], netilmicin [25,39,42,48,58,66], kanamycin $[45,47,73,84,85]$, sisomicin [25,46,48,68,75], neamine [24], gentamicin C1a [6,24], astromicin [25], dihydrostreptomycin [27], dibekacin [28,33,62], gentamicin [59], neomycin [16,77], naphthalene [65], anthracene [67], streptomycin [72], quinoxaline [79], kanamycin B [81], and deuterated paromomycin acetic acid [6]. Of the internal standards used, 13 were aminoglycosides, 3 were modified aminoglycosides $[6,22,81]$, and 4 were not aminoglycosides or congeners of aminoglycosides $[24,65,67,79]$.

\section{Mode of elution}

Although the mobile phase is not directly responsible for chromatographic separation, it can affect chromatographic resolution, selectivity, and efficiency [96]. The selection of a suitable mobile phase for the HPLC system is dependent on the physicochemical properties of the analyte [98]. Since reversed-phase chromatography was reported by most articles, mobile phase solvents used consisted of an aqueous buffer and a non-ultraviolet active water-miscible organic solvent [96]. The solvents included acetonitrile for the separation of netilmicin, gentamicin, and amikacin $[21,39,40,47,60]$; methanol and water mixture for the separation of gentamicin, amikacin, tobramycin, netilmicin, dibekacin, and sisomicin $[20,22,24,36,44]$; and heptanesulphonic acid for separation of gentamicin, and netilmicin [58,59]. Other solvents such as tetrahydrofuran for the separation of amikacin [15]; octanesulphonate for the separation of tobramycin, sisomicin, and netilmicin [48]; and ethylene glycol for the separation of sisomicin [54] were also reported. In the case where ionisable analytes were present, the $\mathrm{pH}$ of the mobile phase had a significant effect on the ionization state(s) of the analyte(s), which eventually affected resolution. Thus, the buffer reported by 
most articles were aqueous, which included tris buffer [23], sodium acetate [24], and potassium phosphate buffer [15]. Basic analytes such as aminoglycosides are protonated at low pH when ionized.

Poor elution of analytes could contribute to peak broadening [96,99]. Out of 71 articles reviewed, 50 employed isocratic elution for the separation of streptomycin, gentamicin, amikacin, dihydrostreptomycin, kanamycin, netilmicin, isepamicin, sisomicin, paromomycin, neomycin, tobramycin, etimicin, and dibekacin; whilst the remaining 21 used gradient elution for the separation of streptomycin, gentamicin, amikacin, dihydrostreptomycin, kanamycin, netilmicin, apramycin, isepamicin, sisomicin, paromomycin, geneticin, neomycin, tobramycin, arbekacin, spectinomycin, hygromycin, dibekacin, astromicin, and micronomicin. For complex multicomponent samples, gradient elution is used since all components cannot be eluted between the retention factor of 1 and 10. An isocratic mode is sufficient if the measured ratio is less than 0.25 , however, if the ratio is greater than 0.25 , then an elution gradient is deemed suitable $[96,99]$.

\section{Derivatization and mode of detection}

Of the 71 articles reviewed, fluorescence (36 articles), UV (15 articles), mass spectrometry (15 articles), resonance Rayleigh scattering (1 article) [32], pulsed electrochemical (1 article) [15], chemiluminescence (1 article) [71], and evaporative light scattering (1 article) [70] were used in the detection of aminoglycosides.

Fluorescence ( $\sim 50 \%)$ was the most used method for the detection of streptomycin, gentamicin, amikacin, dihydrostreptomycin, kanamycin, netilmicin, isepamicin, sisomicin, neomycin, tobramycin, dibekacin, astromicin, and micronomicin. However, non-fluorescence drugs such as aminoglycosides are mostly difficult to detect by this mode due to the absence of a fluorophore. This shortcoming can be mitigated by derivatization [100]. Additionally, the mobile phase used must be selected with care, as highly polar solvents or halide ions can quench fluorescence. It is noteworthy that fluorescence is mostly preferred to UV detection due to its high sensitivity and selectivity [101]. Aminoglycosides assayed in various studies with fluorescence detectors were achieved at an excitation wavelength range of $220 \mathrm{~nm}$ [21] to $490 \mathrm{~nm}$ [77] and emission wavelength range of $415 \mathrm{~nm}$ [57] to $531 \mathrm{~nm}$ [74].

UV-visible detectors are not easily influenced by the mobile phase and surrounding temperature [102]. UV-visible detectors interact with compounds containing chromophores. Since aminoglycosides do not have chromophores, derivatization of these compounds is necessary for their detection. UV-visible was used for the detection of gentamicin, amikacin, tobramycin, streptomycin, netilmicin, and geneticin $[6,27,29,38,39,41,45,47,58,64,65,67]$. In this review, the UV wavelength range used for the detection of aminoglycosides was $195 \mathrm{~nm}[27]$ to $365 \mathrm{~nm}[6,38,45,64]$.

In all the 51 articles that used fluorescence or UV detection, only 3 articles did not use derivatization for the detection of streptomycin, dihydrostreptomycin, and sisomicin [27,30,54]. The most common derivatizing agent used in the bioanalysis of aminoglycosides was o-phthalaldehyde; reported by 24 articles for the derivatization of gentamicin, amikacin, kanamycin, netilmicin, isepamicin, sisomicin, neomycin, tobramycin, dibekacin, astromicin, and micronomicin $[6,14,20,22-26,28,36,37,43,44,48,50,52,53,55,57-$ $59,61,62,66]$. Dansyl chloride for the derivatization of netilmicin [21]; 1-fluoro-2,4-dinitrobenzene for the derivatization of geneticin, gentamicin, and amikacin [29,38,41,45,56,64]; 7-fluoro-4-nitrobenz-2-oxa-1,3diazole for the derivatization of amikacin [74]; benzene sulphonyl chloride for the derivatization of gentamicin [39]; 6-aminoquinolyl-N-hydroxysucciminidylcarbamate for the derivatization of isepamicin [76]; fluorescamine for the derivatization of gentamicin, and tobramycin [40,80]; 1-naphthyl isothiocyanate for the derivatization of amikacin, and tobramycin [65,67]; fluorescein isothiocyanate for the derivatization of tobramycin [77]; o-phthalicdicarboxaldehyde for the derivatization of gentamicin [42]; 2,4,6trinitrobenzene sulfonic acid for the derivatization of tobramycin, and amikacin $[46,47] ; \beta$-naphthoquinone- 
4-sulfonate for the derivatization of streptomycin [49]; ninhydrin for the derivatization of streptomycin [51]; and 9-fluorenylmethylchloroformate for the derivatization of gentamicin, neomycin, netilmicin, sisomicin, isepamicin, and amikacin $[16,60,63,78,82]$ were other derivatizing agents used in the bioanalysis of aminoglycosides.

Challenges associated with UV and fluorescence detections (need for chromophore or fluorophore necessitating derivatization) can be circumvented using mass spectrometry. Also, mass spectrometry can analyze small sample volumes with high precision, sensitivity, and selectivity [68,81]. In this review, 15 articles reported the use of mass spectrometry as a detector for the bioanalysis of neomycin, streptomycin, dihydrostreptomycin, amikacin, kanamycin, paromomycin, tobramycin, spectinomycin, apramycin, hygromycin, gentamicin, and arbekacin [5,31,33-35,68,69,72,73,75,79,81,83-85]. Interestingly, articles published between 1977 [20] to 2002 [67] were dominated by fluorescence or UV detection methods. More importantly, mass spectrometry was the mostly used detection mode from 2014 to 2020 $[34,35,81,83-85]$, except in one case [82]. This is not surprising as mass spectrometry appears to be an effective detection method [103].

In this review, HPLC coupled with resonance Rayleigh scattering detection was used in analyzing three aminoglycosides; amikacin, netilmicin, and etimicin [32]. An advantage of the resonance Rayleigh scattering detector over other spectroscopic techniques is that the detection limit is lower by several orders of magnitude [104].

The pulsed electrochemical detector is mostly used to analyze carbohydrates and polyalcohol. They are also used in analyzing amines, amino acids, and sulphur-containing compounds [105, 106]. One (1) article reported the use of HPLC coupled with the pulsed electrochemical detector in the bioanalysis of amikacin [15]. This approach was used to address the shortfall of using derivatization for the detection of aminoglycosides [15].

Chemiluminescence allows the detection of analytes at ultra-high sensitivity. In this review, 1 article reported the use of chemiluminescence for the detection of amikacin, and the chemiluminescence reagent used was luminol in combination with hydrogen peroxide and $\mathrm{Cu}^{2+}[71]$.

HPLC coupled with evaporative light scattering detector is rapidly becoming a quasi-universal detector, mitigating the need for derivatization of non-absorbing analytes. In this review, one article reported the use of an evaporative light scattering detector for the direct determination of tobramycin [70].

\section{Performance metrics}

Performance metrics are quantifiable terms that indicate the quality of an analytical process. Some performance metrics include specificity, sensitivity, linearity, the lower limit of quantification (LLOQ), limit of detection (LOD), precision, accuracy, and calibration range. In this review, all the 71 articles reported some aspects of method performance characteristics. Method performance characteristics reported by most articles included calibration range, linearity, recovery, repeatability, and intermediate precision (Table 2). Out of the 71 articles reviewed, only four reported on matrix effect $[63,83-85]$. Resolution was reported by one article [75].

The calibration range is often obtained from a calibration curve [107]. Sixty-seven (67) articles reported calibration ranges, whilst 4 did not $[5,23,59,79]$ (Table 2). These calibration ranges of HPLC varied significantly for the various drugs assayed, with some in the range of $0.1-0.5 \mathrm{ng} / \mathrm{mL}$ [14] and others 1250 $200000 \mathrm{ng} / \mathrm{mL}$ [83]. Of the 20 aminoglycosides reported, only eight had established a therapeutic reference range. The reported calibration range of some of the analytes reported was outside the established 
therapeutic reference range $[14,15,24,25,30,31,34,37,48,55,57,61,65,67,71,74,75,80-82]$, which invalidates the measurements obtained from the bioanalysis.

The quality of a bioanalytical method is highly dependent on the linearity of the calibration curve [108]. The linearity of the calibration curve is usually expressed as a coefficient of correlation $\left(R^{2}\right)$. The coefficient of correlation close to $1\left(R^{2} \approx 1\right)$ is mostly considered ideal. In the current review, 61 articles reported $R^{2}$ values for almost all analytes under consideration, whilst 10 articles did not $[23,28,33,39,45,47,50,55,56,84]$. The reported coefficient of correlation was between 0.964 [75] and 1.0 $[48,60,76,82]$. Additionally, $y$-intercept, the slope of the regression of line, and residual sum of squares can also be used in evaluating linearity [109]. Out of 71 articles reviewed, 39 articles reported values for both the slope of regression and the y-intercept.

Accuracy of a bioanalytical method is normally expressed as the percent recovery by the assay of the known added amount of analyte. In this study, 63 articles reported percent recoveries that ranged mostly between $61.0 \%$ [72] to $114.0 \%$ [5]. Two (2) articles reported recoveries of $4.9 \%$ [28] and $36 \%$ [76], respectively.

LLOQ is defined as the amount of analyte in a biological matrix that can be quantitatively determined with suitable precision and accuracy [110]. Thirty-six (36) articles reported LLOQ for aminoglycosides assayed between $1 \mathrm{ng} / \mathrm{mL}$ [72] to $2340 \mathrm{ng} / \mathrm{mL}$ [79]. Forty-one (41) articles also reported LOD values in the range $0.3 \mathrm{ng} / \mathrm{mL}$ [61] to $75000 \mathrm{ng} / \mathrm{ml}$ [56]. There were instances where the same article reported both the LLOQ and LOD.

Repeatability expresses the closeness of results obtained with the same sample using the same procedure, operators, measuring system, operating conditions, and location over a short period of time. Out of 71 articles, 58 reported repeatability values ranging from $0.28 \%$ [69] to $36 \%$ [30]. Intermediate precision also refers to laboratory variations such as different days, instruments, and analyses. Out of the 71 reviewed articles, 56 reported intermediate precision values ranging between $0.331 \%$ [80] to $19.76 \%$ [52], which is within the acceptable limit of $20 \%$ [111]. 
Table 2. Performance metrics of HPLC method for bioanalysis of aminoglycosides.

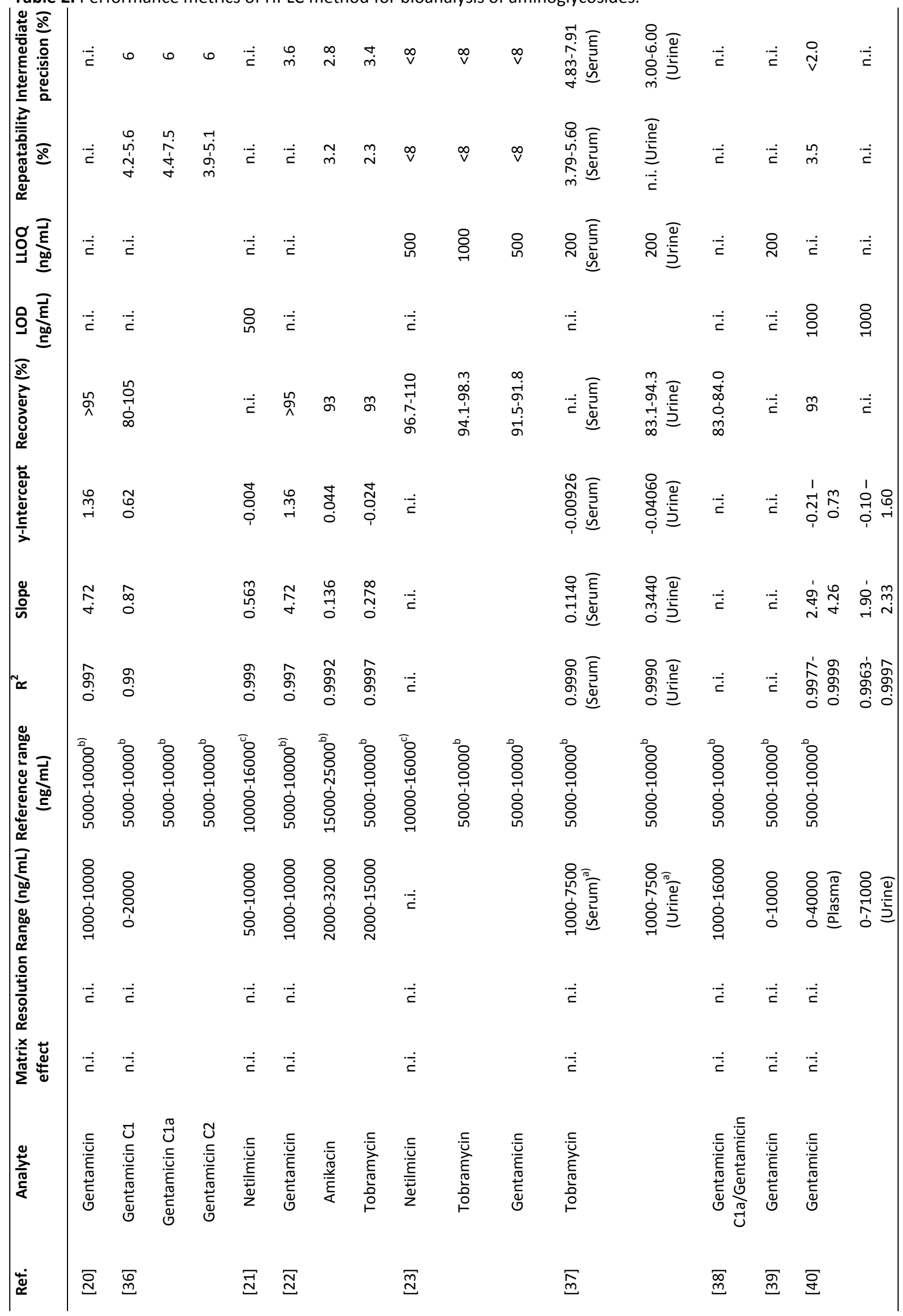


Table 2. Continued...

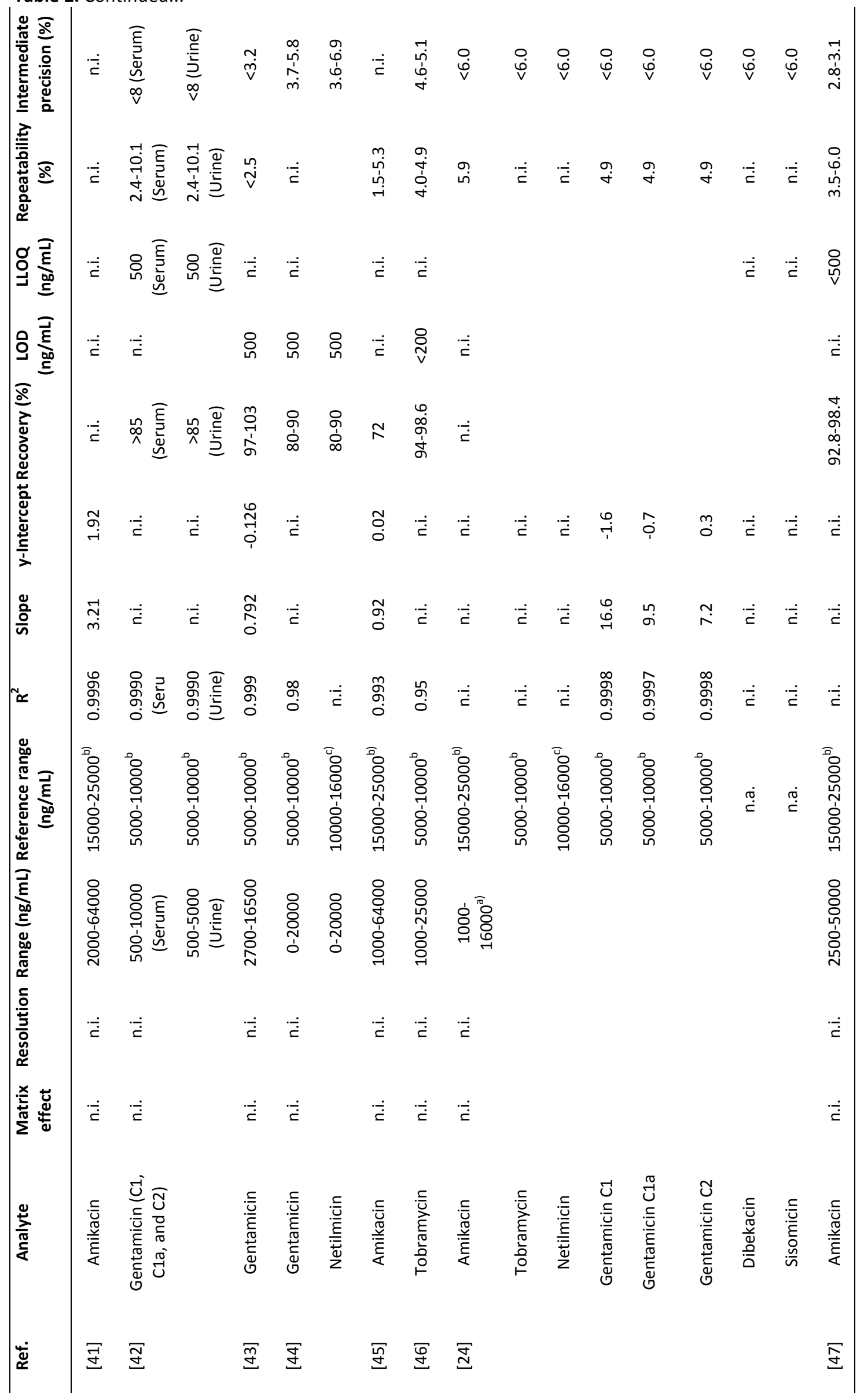


Table 2. Continued...

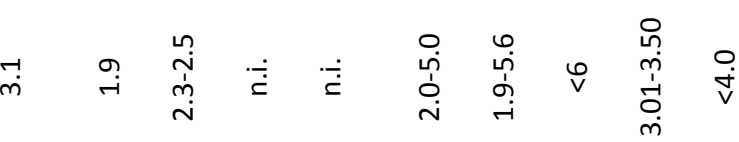

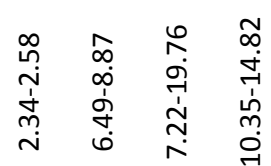
焉

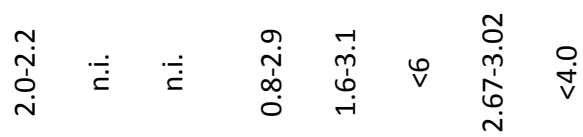

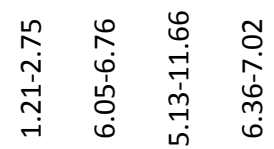
g $\underset{\exists}{\stackrel{\bar{\xi}}{\underline{\underline{g}}}}$

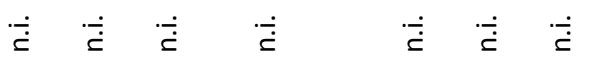
$\ddot{\dot{\varepsilon}} \dot{\dot{\varepsilon}}$

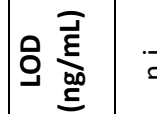
\&
ஓ ㅇ

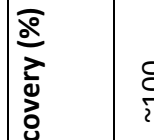

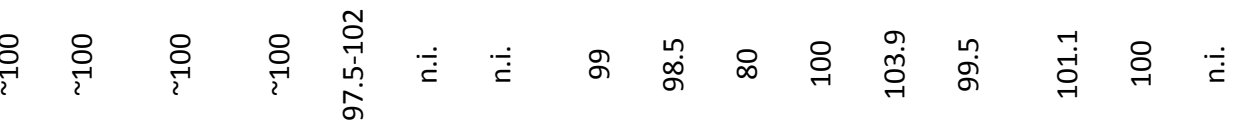

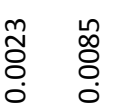

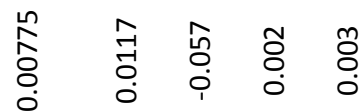
$\begin{array}{lllll}0 & 1 & 0 & 1 & 0 \\ 0 & 0 & 0 & 0 & 0 \\ 0 & 0 & 0 & 0\end{array}$

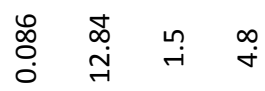

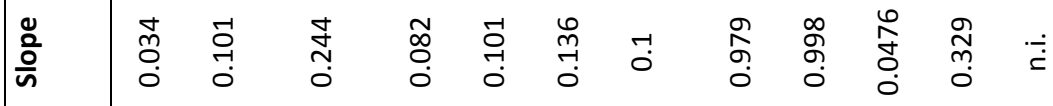

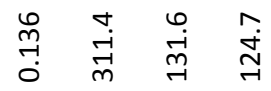

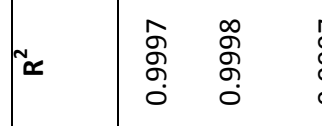
命 :

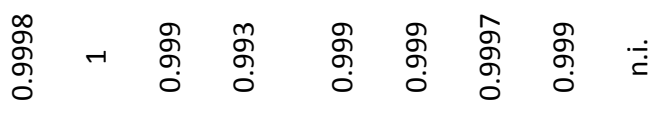

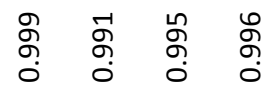

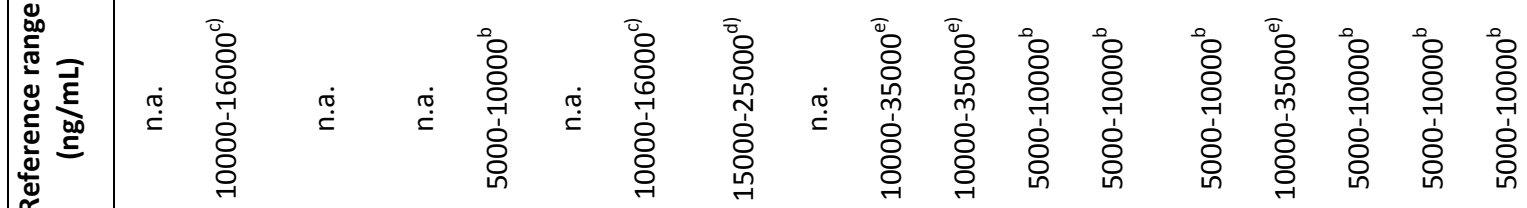

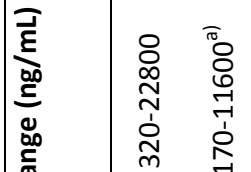

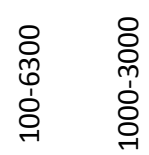

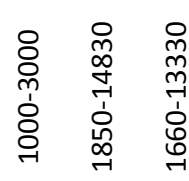

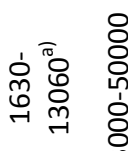

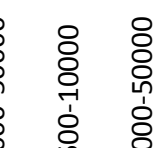

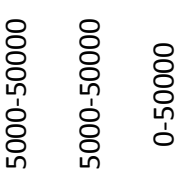

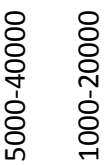
)
$\stackrel{\dot{\varepsilon}}{\dot{\varepsilon}}$
$\stackrel{\dot{E}}{\dot{E}} \quad \dot{\dot{E}} \quad \dot{\dot{E}}$

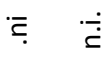
焉蓄
$\stackrel{\dot{\varepsilon}}{\dot{\varepsilon}}$

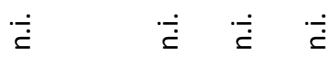
$\stackrel{\dot{\varepsilon}}{\dot{E}}$

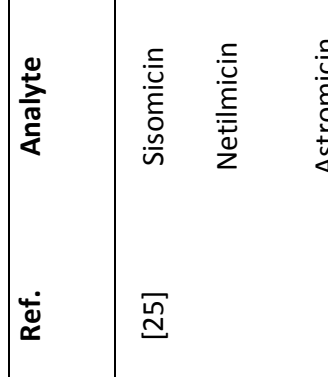
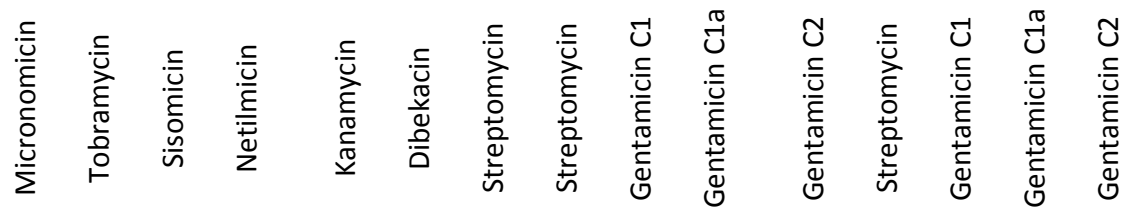
$\stackrel{\infty}{\stackrel{\infty}{\infty}}$

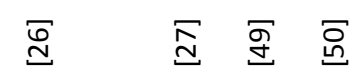
크 กี่ 
Table 2. Continued...

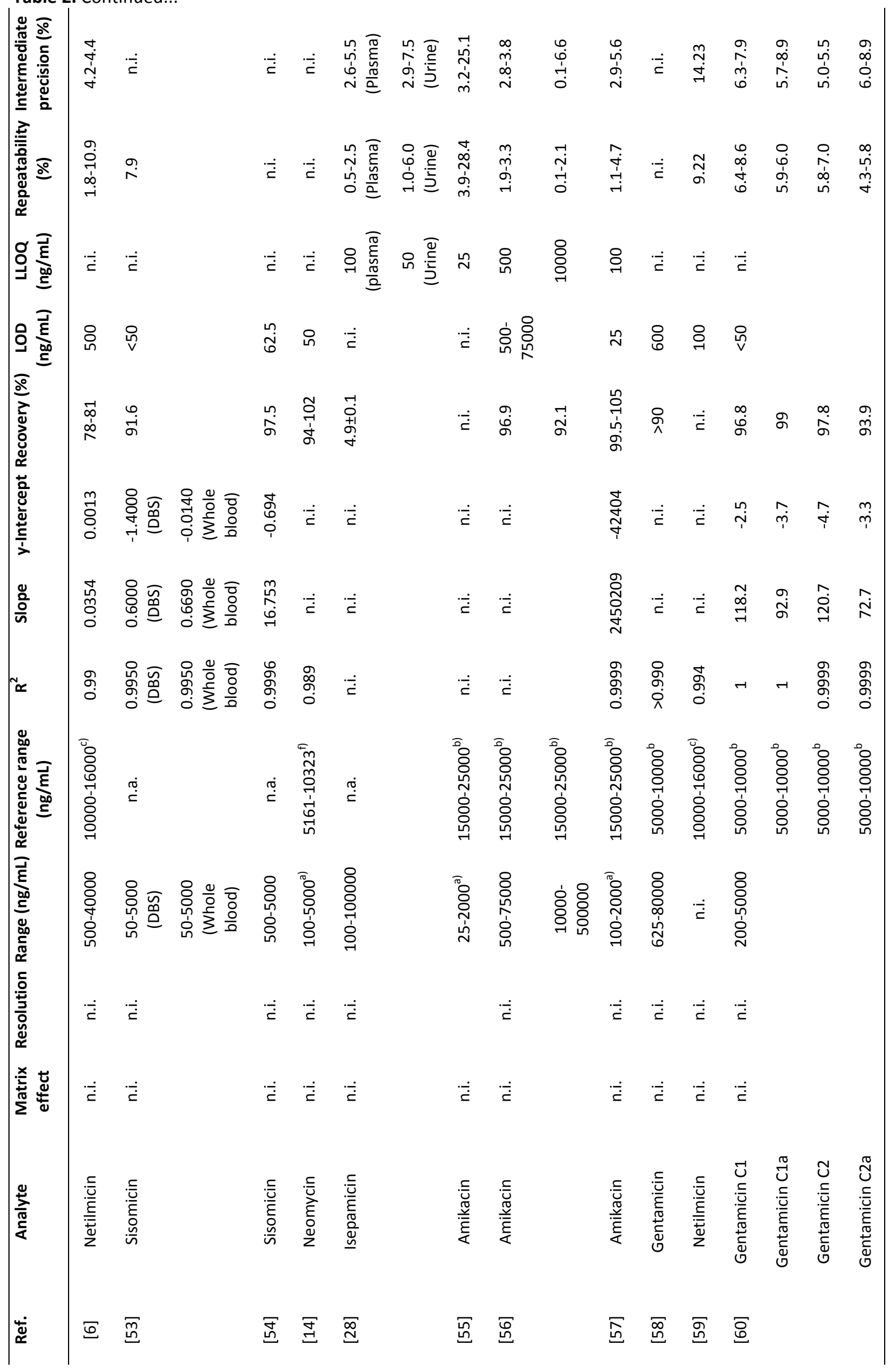


Table 2. Continued...

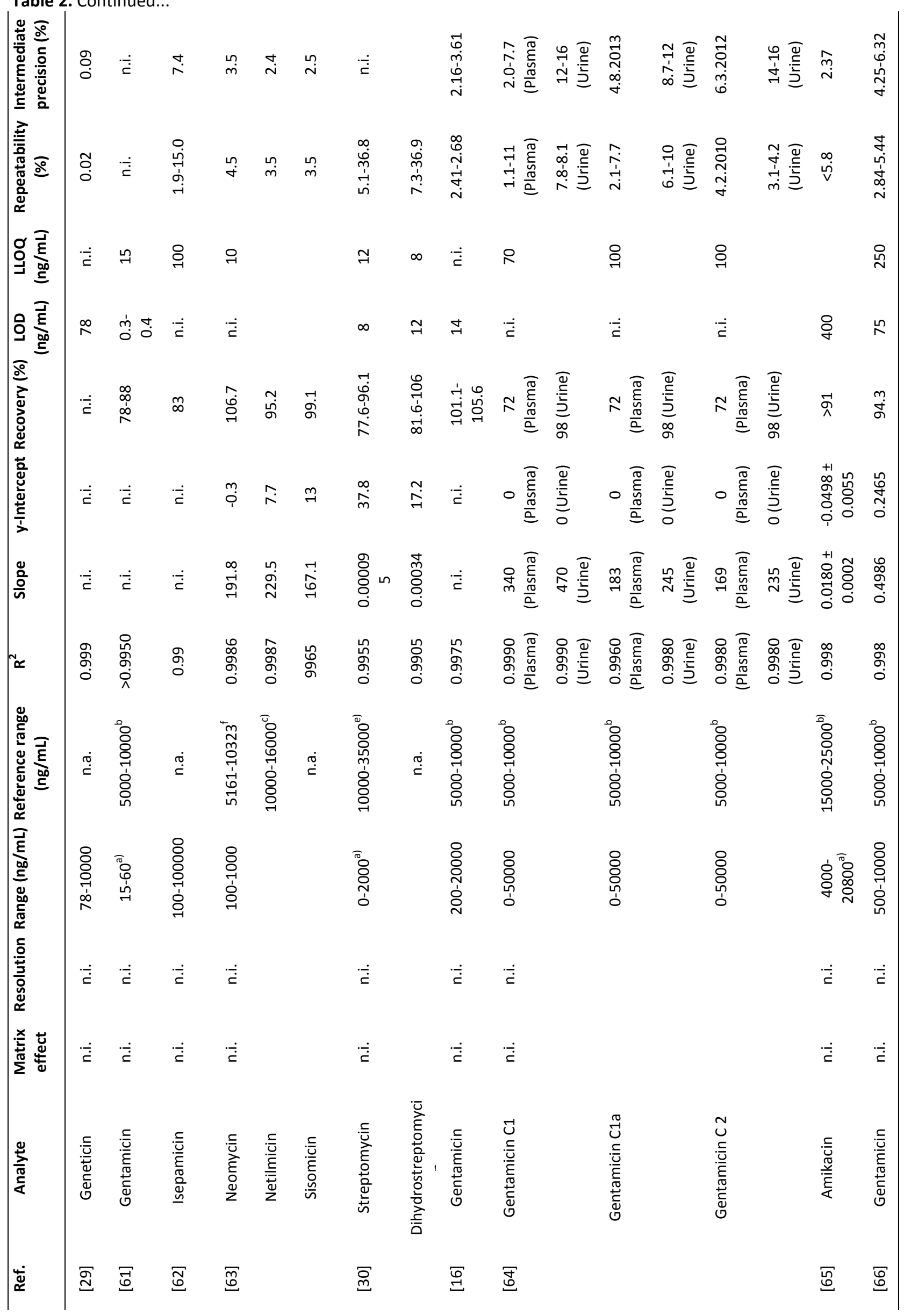


Table 2. Continued...

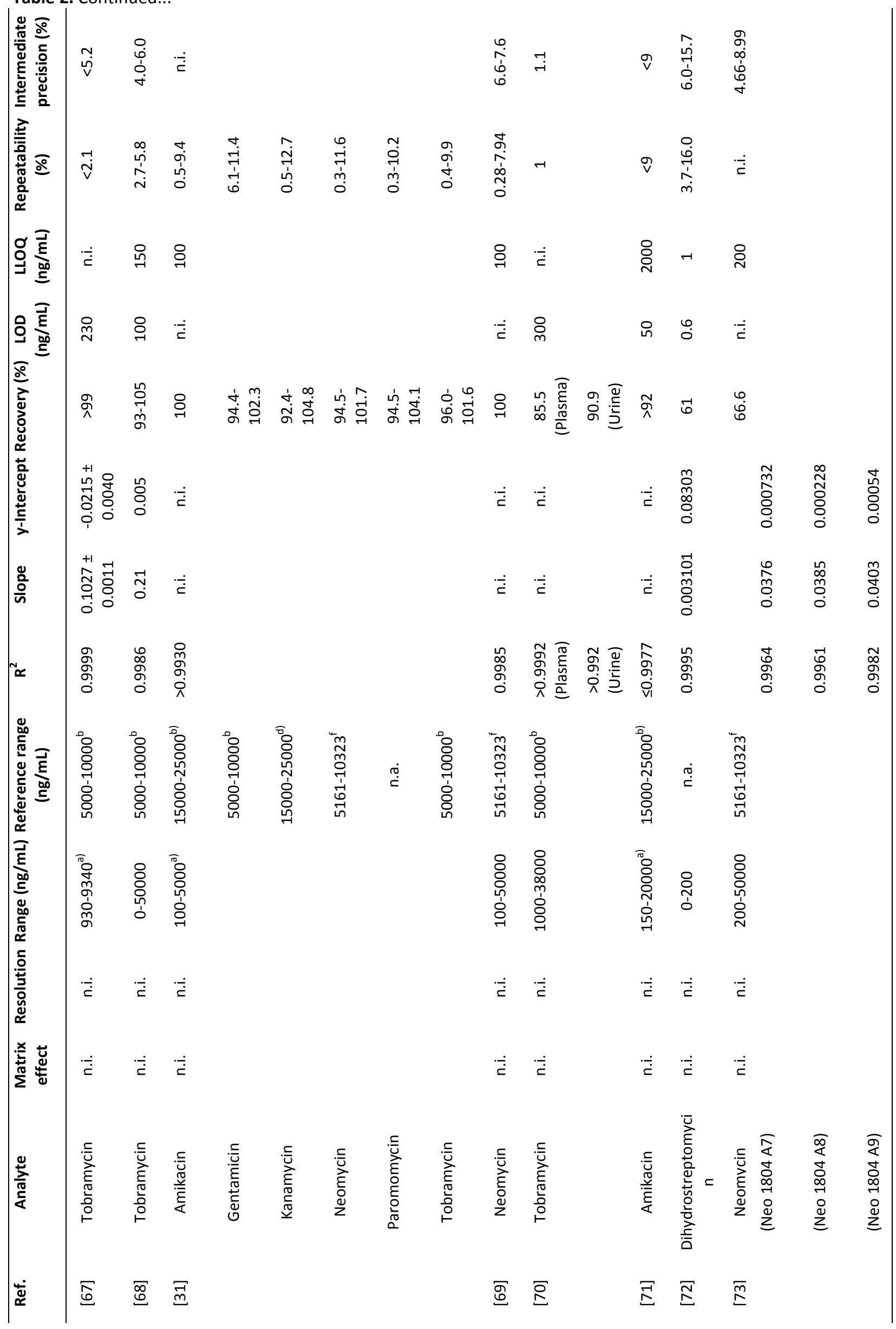


Table 2. Continued...

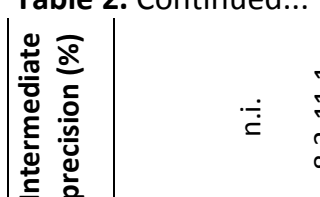

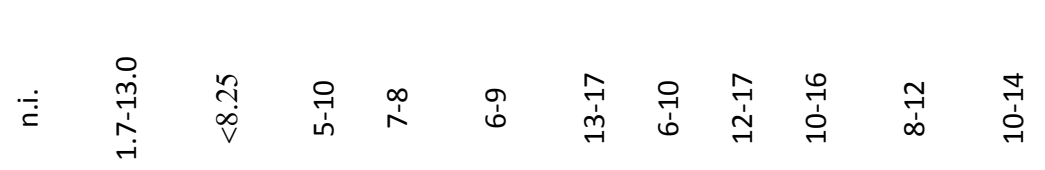

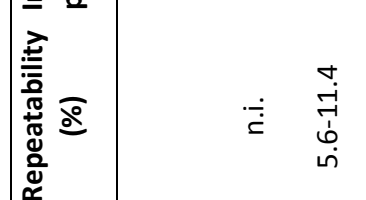

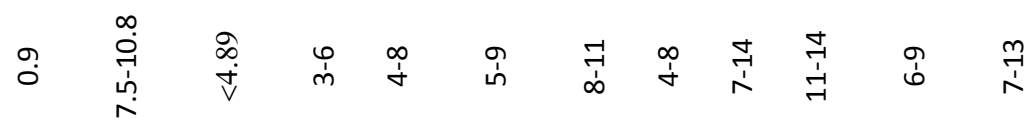

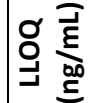

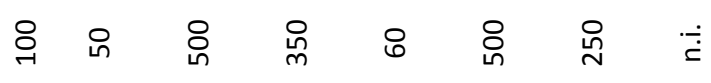

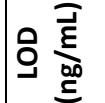

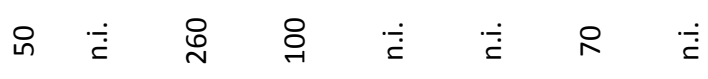

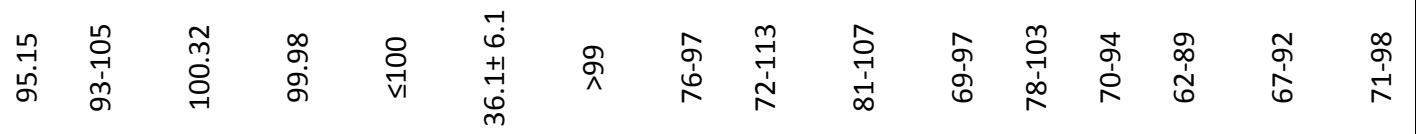

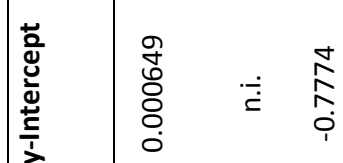

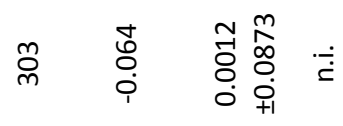

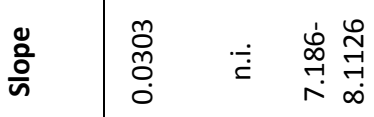

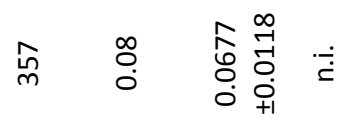
$\approx$ \%ิ

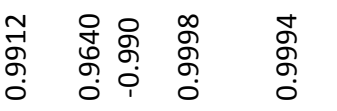

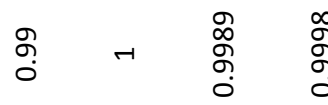

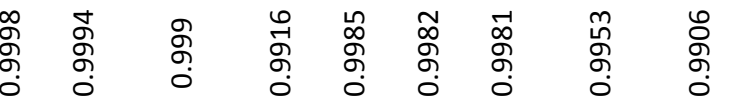

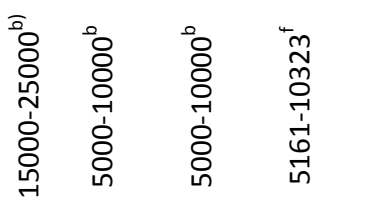

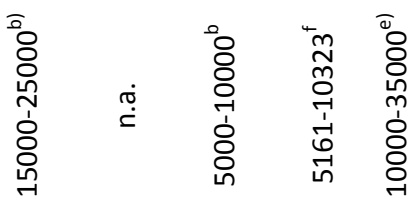

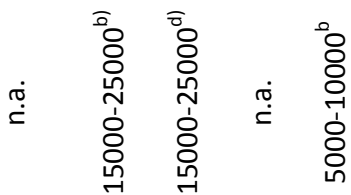

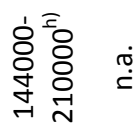

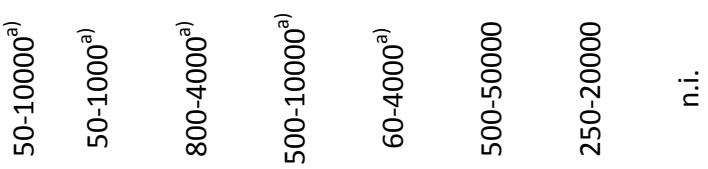

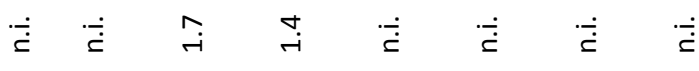
$\dot{\vec{E}} \dot{\dot{E}}$

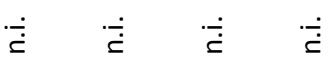

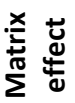

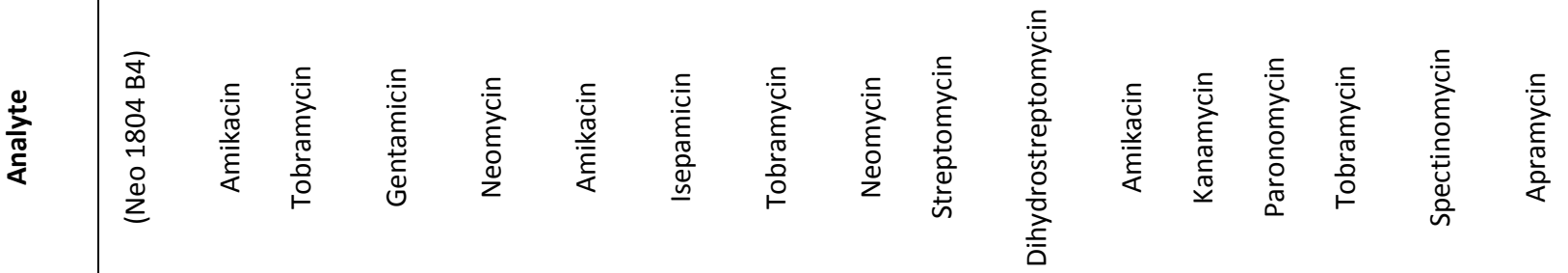
$\stackrel{\Xi}{\Xi}$
$\stackrel{\Re}{\Xi} \underset{\Xi}{\Xi}$ 
Table 2. Continued...

\begin{tabular}{|c|c|c|c|c|c|c|c|c|c|c|c|c|c|c|c|c|c|}
\hline 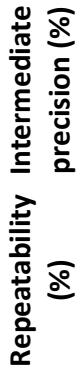 & $\begin{array}{l}\text { ㄱ } \\
\text { ò }\end{array}$ & 움 & 움 & 움 & 오 & $\begin{array}{l}\text { ํㅜ } \\
\stackrel{\text { - }}{\mathrm{V}}\end{array}$ & & $\begin{array}{l}\infty \\
\dot{v} \\
v\end{array}$ & $\stackrel{\infty}{\dot{\forall}}$ & 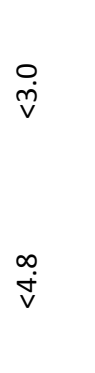 & $\dot{\dot{\Sigma}}$ & 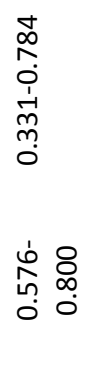 & 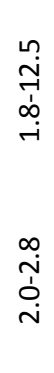 & 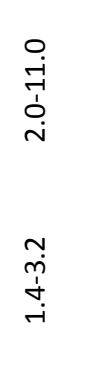 & $\begin{array}{l}\infty \\
0 \\
\rightarrow \\
\dot{b} \\
\dot{v}\end{array}$ & 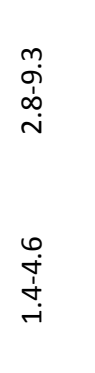 & $\begin{array}{l}m \\
\infty \\
\infty \\
o \\
0 \\
0\end{array}$ \\
\hline o & & & & & ㅇํㅁ & 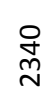 & ஜூలి & $\dot{\dot{\dot{x}}}$ & & & 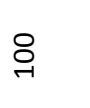 & $\underset{\sigma}{n}$ & $\dot{\dot{\dot{g}}}$ & & & & 유 \\
\hline 오 & & & & & ঃ & ㅇํํ & 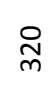 & $\stackrel{\infty}{\rightarrow}$ & $\vec{\sim}$ & 느ํ & $\dot{\dot{E}}$ & $\begin{array}{l}\text { mे } \\
\text { in }\end{array}$ & $\dot{\dot{E}}$ & & & & $\stackrel{\sim}{\text { v }}$ \\
\hline 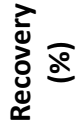 & $\begin{array}{l}\infty \\
\text { के } \\
\infty\end{array}$ & $\begin{array}{l}\text { Oิ } \\
\stackrel{-}{N} \\
\infty\end{array}$ & $\begin{array}{l}\stackrel{+}{-1} \\
\stackrel{1}{0} \\
\stackrel{0}{1}\end{array}$ & $\begin{array}{l}\text { 음 } \\
\text { ํํํ }\end{array}$ & 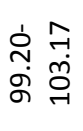 & ஸ் & $\begin{array}{l}\varphi \\
\ddot{\infty} \\
\infty\end{array}$ & ஸ்' ஜே & 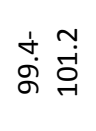 & 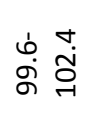 & 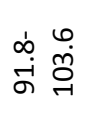 & $\begin{array}{l}m \\
m \\
m \\
\infty \\
\infty\end{array}$ & ํํำ & ণ્ণ & 옥 & ث̊ํㅁ & $\infty$ \\
\hline 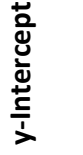 & & & & & 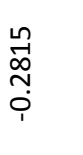 & $\dot{\dot{C}}$ & & $\begin{array}{c}\text { Oి } \\
\text { mె } \\
\text { i }\end{array}$ & $\begin{array}{l}\mathscr{V} \\
0 \\
\dot{m}\end{array}$ & $\begin{array}{l}\text { ठ̀ } \\
\text { O } \\
-i\end{array}$ & $\begin{array}{l}\text { 넛 } \\
\text { ○ } \\
0\end{array}$ & $\begin{array}{l}\text { గ్ర } \\
\text { ○ุ } \\
\text { - }\end{array}$ & $\dot{\dot{E}}$ & & & & $\dot{\dot{\check{C}}}$ \\
\hline$\frac{\text { 음 }}{\text { 는 }}$ & & & & & 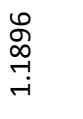 & $\dot{\dot{c}}$ & & $\begin{array}{l}\emptyset \\
\dot{0} \\
\dot{\infty} \\
\stackrel{\leftrightarrow}{\sim}\end{array}$ & 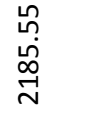 & 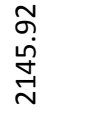 & $\begin{array}{c}\frac{n}{\sigma} \\
\stackrel{0}{0}\end{array}$ & 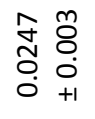 & $\stackrel{\dot{\dot{E}}}{ }$ & & & & $\dot{\dot{\check{C}}}$ \\
\hline $\mathbb{N}^{\infty}$ & $\begin{array}{l}\infty \\
\text { ने } \\
\text { ब़े }\end{array}$ & $\begin{array}{l}\text { ूু } \\
\text { ऴ }\end{array}$ & $\begin{array}{l}\text { ळू } \\
\text { О़ } \\
0\end{array}$ & $\begin{array}{l}\hat{\phi} \\
\text { ুே }\end{array}$ & $\begin{array}{l}\text { ळे } \\
\text { ऴ }\end{array}$ & $\begin{array}{l}\infty \\
\text { Оా } \\
\circ\end{array}$ & $\begin{array}{l}\infty \\
\text { ळ̆ } \\
0\end{array}$ & $\begin{array}{l}\text { ŏ } \\
\text { ने }\end{array}$ & बे & बे & $\stackrel{\dot{E}}{\dot{E}}$ & જ̆ & $\begin{array}{l}\text { Оे } \\
\text { Оू }\end{array}$ & $\begin{array}{l}\infty \\
\infty \\
\text { Oे } \\
0\end{array}$ & $\begin{array}{l}\text { ूे } \\
\text { Оे }\end{array}$ & $\begin{array}{l}\text { ठे } \\
\text { बे }\end{array}$ & $\begin{array}{l}\text { Оे } \\
\text { Оे } \\
\text { ○े }\end{array}$ \\
\hline 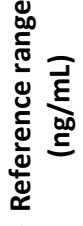 & 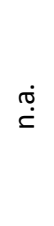 & $\begin{array}{l}8 \\
8 \\
8 \\
8 \\
\text { ㅁ } \\
8 \\
8 \\
\text { ㅇ }\end{array}$ & 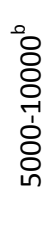 & 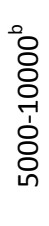 & 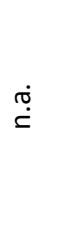 & 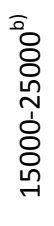 & 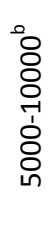 & 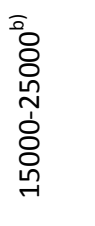 & $\begin{array}{l}\overline{0} \\
8 \\
8 \\
6 \\
-1 \\
8 \\
8 \\
8\end{array}$ & 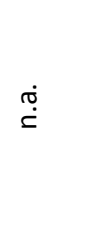 & 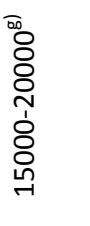 & 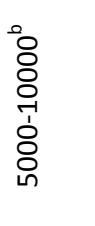 & 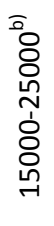 & 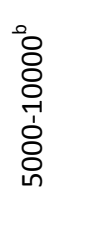 & 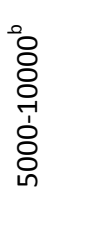 & 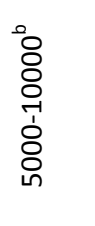 & 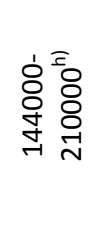 \\
\hline 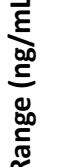 & & & & & 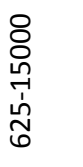 & $\dot{\dot{E}}$ & & $\begin{array}{l}8 \\
\varnothing \\
\infty \\
\text { ஸे }\end{array}$ & \begin{tabular}{l}
8 \\
$o$ \\
\multirow{+}{*}{} \\
ô
\end{tabular} & $\begin{array}{l}8 \\
\varnothing \\
\\
0 \\
\grave{1}\end{array}$ & 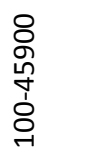 & 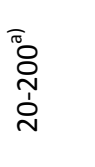 & 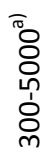 & ঠ் ઠ & ㅎํㅇ ઠ & ঠ் ઠ & 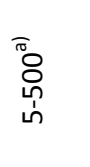 \\
\hline 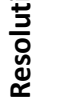 & & & & & $\dot{\dot{\Sigma}}$ & $\dot{\dot{\Sigma}}$ & & $\dot{\dot{E}}$ & & & $\dot{\dot{\dot{E}}}$ & $\dot{\dot{\dot{E}}}$ & $\dot{\dot{\Sigma}}$ & & & & $\dot{\dot{I}}$ \\
\hline 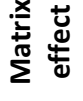 & & & & & $\dot{\dot{E}}$ & $\dot{\dot{E}}$ & & $\dot{\dot{E}}$ & & & 1 & $\dot{\dot{\epsilon}}$ & $\dot{\dot{E}}$ & & & & $\stackrel{\dot{C}}{\dot{C}}$ \\
\hline 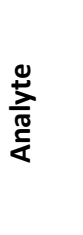 & 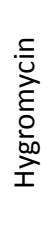 & 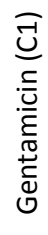 & 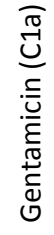 & 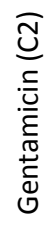 & 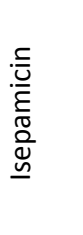 & 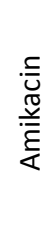 & 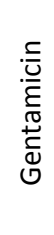 & 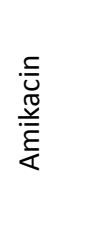 & 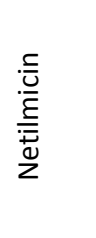 & & $\begin{array}{l}. \frac{5}{0} \\
\frac{\pi}{\pi} \\
\frac{\pi}{0} \\
\frac{0}{4}\end{array}$ & 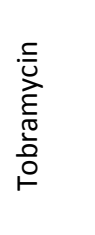 & 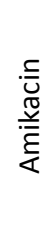 & 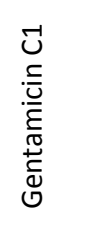 & 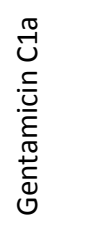 & 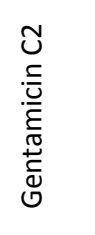 & 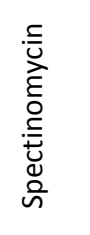 \\
\hline & & & & & $\stackrel{\infty}{\unrhd}$ & న & & $\underset{\widetilde{m}}{\tilde{m}}$ & & & $\stackrel{\bar{m}}{\stackrel{m}{n}}$ & $\stackrel{\square}{\infty}$ & $\underset{\infty}{\not}$ & & & & 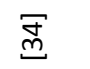 \\
\hline
\end{tabular}


Table 2. Continued...

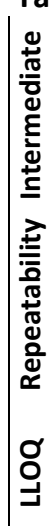
a connueat.

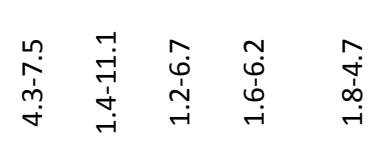

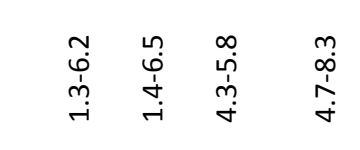

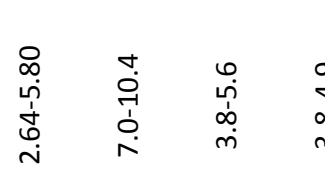

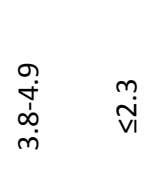

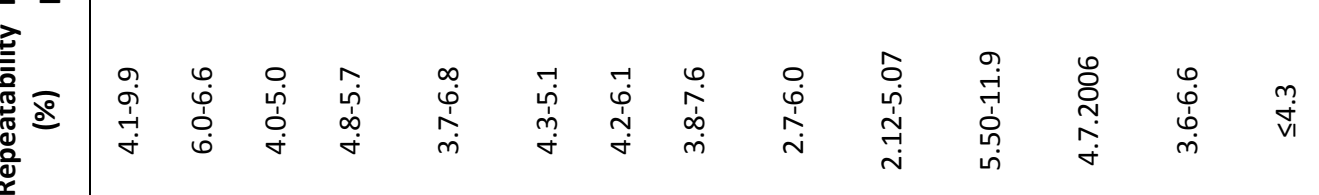

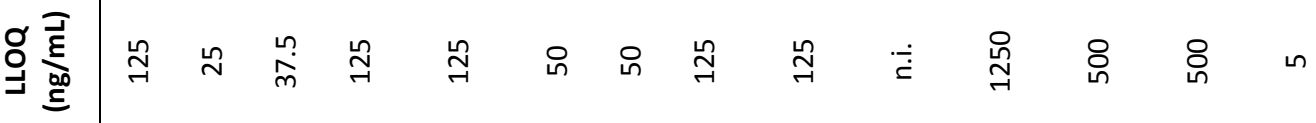
올

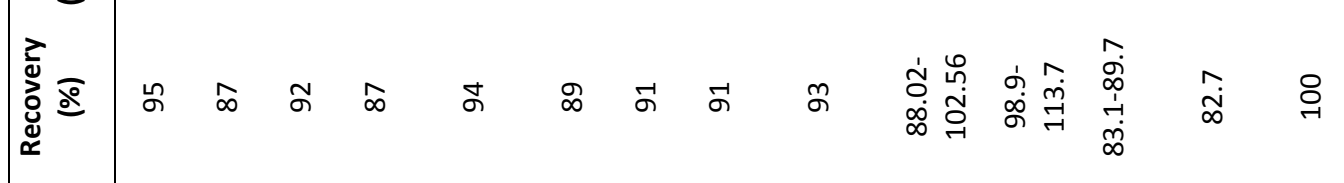

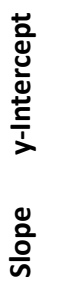

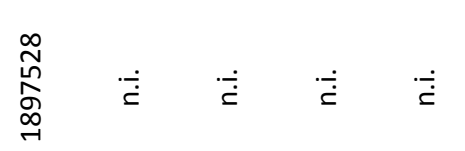

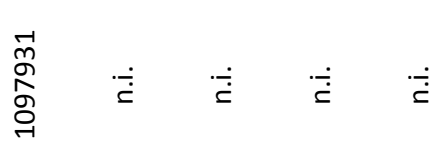

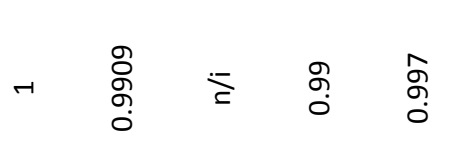

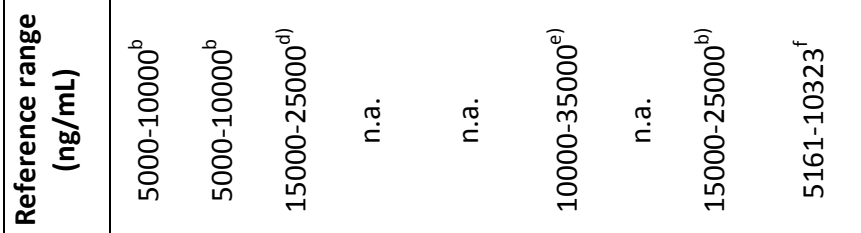

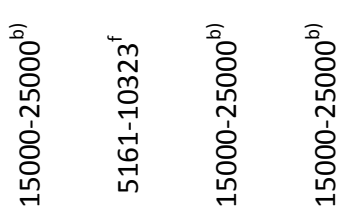

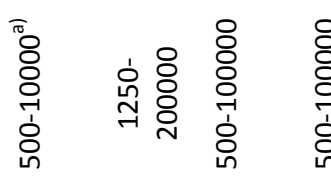
8
0
0
0

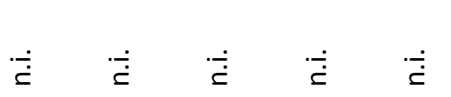

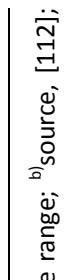$$
\text { (n) }
$$

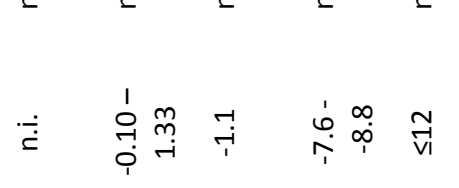

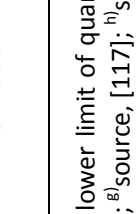

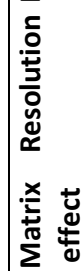

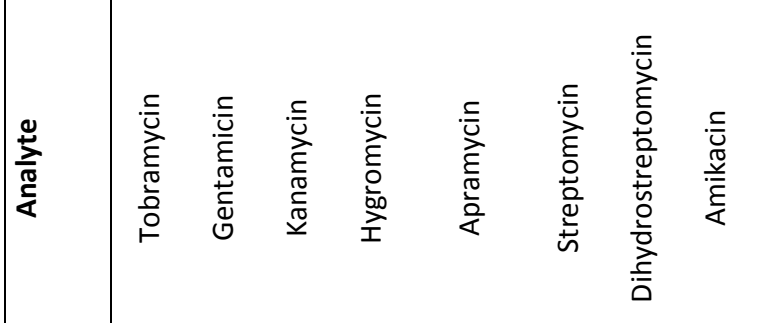
$\ddot{\ddot{\varpi}}$ 


\section{Conclusion and outlook}

Despite reported nephrotoxic and ototoxic potentials of aminoglycosides, their use in clinical settings remains relevant. The current study sought to review bioanalytical methods (specifically liquid chromatography) used in the assay of aminoglycosides in biological matrices. In all, 71 articles were reviewed, and 66 of these articles reported the use of reversed-phase liquid chromatography as a bioanalytical method $[7,119]$.

The commonest sample treatment procedures adopted in the analysis of aminoglycosides using HPLC were protein precipitation (50\%) and solid phase extraction (39\%). Surprisingly, none of the current sample preparation methods was used by any of the articles reported in this review. It will be interesting if recent sample preparation methods such as solid-phase microextraction, micro-solid-phase extraction, dispersive micro-solid-phase extraction, magnetic solid-phase extraction, microextraction by packed sorbent, stir bar sorptive extraction, spin column extraction, liquid-phase microextraction, single-drop microextraction, hollow fiber liquid-phase microextraction, dispersive liquid-liquid microextraction, molecularly imprinted solid-phase extraction, and molecularly imprinted solid-phase micro-extraction [120122] could be applied for bioanalysis of aminoglycosides with the potential of improving method sensitivity and selectivity.

Fluorescence (50\%), UV (24\%), and mass spectrometry (21\%) were the most adopted mode of detection in the assay of aminoglycosides, according to this review. Since mass spectrometry has been established as the detection mode of choice for bioanalysis of aminoglycoside using liquid chromatography in recent years, it is strongly recommended for use except in resource-challenged countries where fluorescence or UV detection methods can be applied after derivatization.

There is the need to establish a therapeutic reference range for all the clinically reported 20 aminoglycosides since the calibration range of analytical methods for the bioanalysis of aminoglycosides should cover such a range. It was quite surprising that some of the calibration range was outside the established therapeutic reference range. It is recommended that future liquid chromatography methods for the analysis of aminoglycosides should have calibration ranges covering established reference therapeutic ranges.

Although this review is not a systematic one, the information provided is intended to serve as a comprehensive reference for related research that may involve the assay of aminoglycosides (pharmacokinetic or drug monitoring studies).

Conflict of interest: The authors declare no conflict of interest.

\section{References}

[1] R. Hari, S. Taherunnisa, S.Y. Raut, S. Mutalik, K.B. Koteshwara. Challenges in the development of analytical test procedure for aminoglycosides: A critical review. J. Appl. Pharm. Sci 9 (2019) 145-152. https://doi.org/10.7324/JAPS.2019.91118.

[2] K. Chan, W. Wang, K.R. Ledesma, T. Yin, V.H. Tam. A robust LC-MS/MS method for amikacin: application to cellular uptake and pharmacokinetic studies. Bioanalysis 12 (2020) 445-454. https://doi.org/10.4155/bio-2020-0007.

[3] M.-P. Mingeot-Leclercq, Y. Glupczynski, P.M. Tulkens. Aminoglycosides: activity and resistance. Antimicrobial agents chemotherapy 43 (1999) 727-737. https://doi.org/10.1128/AAC.43.4.727.

[4] L. Šoltés. Aminoglycoside antibiotics-two decades of their HPLC bioanalysis. Biomedical Chromatography 13 (1999) 3-10. https://doi.org/10.1002/(SICI)1099-0801(199902)13:1<3::AIDBMC811>3.0.CO;2-T. 
[5] W.-x. Zhu, J.-z. Yang, W. Wei, Y.-f. Liu, S.-s. Zhang. Simultaneous determination of 13 aminoglycoside residues in foods of animal origin by liquid chromatography-electrospray ionization tandem mass spectrometry with two consecutive solid-phase extraction steps. Journal of Chromatography A 1207 (2008) 29-37. https://doi.org/10.1016/j.chroma.2008.08.033.

[6] S. Dionisotti, F. Bamonte, M. Gamba, E. Ongini. High-performance liquid chromatographic determination of netilmicin in guinea-pig and human serum by fluorodinitrobenzene derivatization with spectrophotometric detection. Journal of Chromatography B: Biomedical Sciences 434 (1988) 169-176. https://doi.org/10.1016/0378-4347(88)80071-9.

[7] A. Cabanes, Y. Cajal, I. Haro, J.G. Anton, F. Reig, M. Arboix. Gentamicin determination in biological fluids by HPLC, using tobramycin as internal standard. Journal of liquid chromatography 14 (1991) 1989-2010. https://doi.org/10.1080/01483919108049669.

[8] S.K. Amponsah, G.O. Adjei, C. Enweronu-Laryea, K.A. Bugyei, K. Hadji-Popovski, J.A.L. Kurtzhals, K. Kristensen. Population Pharmacokinetic Characteristics of Amikacin in Suspected Cases of Neonatal Sepsis in a Low-Resource African Setting: A Prospective Nonrandomized Single-Site Study. Curr Ther Res Clin Exp 84 (2017) e1-e6. https://doi.org/10.1016/j.curtheres.2017.01.001.

[9] S.K. Amponsah, K.F. Opuni, K.A. Antwi, V.P. Kunkpeh. Effect of aminophylline on the pharmacokinetics of amikacin in Sprague-Dawley rats. J Infect Dev Ctries 13 (2019) 251-254. https://doi.org/10.3855/iidc.10514.

[10] S.K. Amponsah, K.F.M. Opuni, A.A. Donkor. Animal model investigation suggests betamethasone alters the pharmacokinetics of amikacin. ADMET and DMPK 6 (2018) 279-283. http://dx.doi.org/10.5599/admet.613.

[11] S.K. Banerjee, A. Wells, A. Dasgupta. Operator error: a critical determinant of false amikacin and tobramycin concentrations using fluorescence polarization immunoassay kits and TDX analyzer. Therapeutic drug monitoring 21 (1999) 540. https://doi.org/10.1097/00007691-199910000-00009.

[12] S.K. Maitra, T.T. Yoshikawa, C.M. Steyn, L.B. Guze, M.C. Schotz. Amikacin assay in serum by highperformance liquid chromatography. Antimicrobial Agents Chemotherapy 14 (1978) 880-885. https://doi.org/10.1128/AAC.14.6.880.

[13] A. Marzo, L. Dal Bo. Chromatography as an analytical tool for selected antibiotic classes: a reappraisal addressed to pharmacokinetic applications. Journal of Chromatography A 812 (1998) 17-34. https://doi.org/10.1016/s0021-9673(98)00282-9.

[14] V.K. Agarwal. High performance liquid chromatographic determination of neomycin in milk using a HISEP column. Journal of liquid chromatography 13 (1990) 2475-2487. https://doi.org/10.1080/01483919008049047.

[15] G. Brajanoski, J. Hoogmartens, K. Allegaert, E. Adams. Determination of amikacin in cerebrospinal fluid by high-performance liquid chromatography with pulsed electrochemical detection. J Chromatogr B Analyt Technol Biomed Life Sci 867 (2008) 149-152. https://doi.org/10.1016/j.jchromb.2008.03.016.

[16] A. Yusuf, S. Al-Rawithi, D. Raines, H. Frayha, A. Toonsi, I. Al-Mohsen, A. El-Yazigi. Simplified highperformance liquid chromatographic method for the determination of gentamicin sulfate in a microsample of plasma: Comparison with fluorescence polarization immunoassay. Therapeutic drug monitoring 21 (1999) 647. https://doi.org/10.1097/00007691-199912000-00012.

[17] Y. Al-Shamalii, Y.M. Ali, R.A. Al-Shamalii, M. Al-Melahi, F.R. Al-Shammari, A. Alsaber, W. Al-Adsani. Don't close the book on tocilizumab for the treatment of severe COVID-19 pneumonia- The jury is still out- The Kuwait experience. PLOS ONE 16 (2021). https://doi.org/10.1371/journal.pone.0254379.

[18] R. Hari, S. Taherunnisa, S.Y. Raut, S. Mutalik, K.B. Koteshwara. Challenges in the development of analytical test procedure for aminoglycosides: A critical review. J. Appl. Pharm. Sci 9 (2019) 145-152. https://doi.org/10.7324/JAPS.2019.91118.

[19] X. Zhang, J. Wang, Q. Wu, L. Li, Y. Wang, H. Yang. Determination of Kanamycin by High Performance Liquid Chromatography. Molecules 24 (2019). https://doi.org/10.3390/molecules24101902. 
[20] J.P. Anhalt. Assay of gentamicin in serum by high-pressure liquid chromatography. Antimicrobial Agents Chemotherapy 11 (1977) 651-655. https://doi.org/10.1128/AAC.11.4.651.

[21] G.W. Peng, G.G. Jackson, W.L. Chiou. High-pressure liquid chromatographic assay of netilmicin in plasma. Antimicrobial Agents Chemotherapy 12 (1977) 707-709. https://doi.org/10.1128/AAC.12.6.707.

[22] J.P. Anhalt, S.D. Brown. High-performance liquid-chromatographic assay of aminoglycoside antibiotics in serum. Clinical chemistry 24 (1978) 1940-1947. https://doi.org/10.1093/clinchem/24.11.1940 .

[23] S.-E. Bäck, I. Nilsson-Ehle, P. Nilsson-Ehle. Chemical assay, involving liquid chromatography, for aminoglycoside antibiotics in serum. Clinical chemistry 25 (1979) 1222-1225. https://doi.org/10.1093/clinchem/25.7.1222.

[24] L. Essers. An automated high-performance liquid chromatographic method for the determination of aminoglycosides in serum using pre-column sample cleanup and derivatization. Journal of Chromatography B: Biomedical Sciences 305 (1984) 345-352. https://doi.org/10.1016/s03784347(00)83348-4.

[25] T. Kawamoto, I. Mashimo, S. Yamauchi, M. Watanabe. Determination of sisomicin, netilmicin, astromicin and micronomicin in serum by high-performance liquid chromatography. Journal of Chromatography B: Biomedical Sciences 305 (1984) 373-379. https://doi.org/10.1016/s03784347(00)83351-4.

[26] H. Kubo, Y. Kobayashi, T. Nishikawa. Rapid method for determination of kanamycin and dibekacin in serum by use of high-pressure liquid chromatography. Antimicrobial Agents Chemotherapy 28 (1985) 521-523. https://doi.org/10.1128/aac.28.4.521.

[27] N. Kurosawa, S. Kuribayashi, E. Owada, K. Ito, M. Nioka, M. Arakawa, R.J.J.o.C.B.B.S. Fukuda, Applications. Determination of streptomycin in serum by high-performance liquid chromatography. Journal of Chromatography B: Biomedical Sciences 343 (1985) 379-385. https://doi.org/10.1016/s0378-4347(00)84606-x.

[28] J.A. Maloney, W.M. Awni. High-performance liquid chromatographic determination of isepamicin in plasma, urine and dialysate. Journal of Chromatography B: Biomedical Sciences 526 (1990) 487-496. https://doi.org/10.1016/s0378-4347(00)82530-x.

[29] C. Bethune, T. Bui, M.L. Liu, M.A. Kay, R.J. Ho. Development of a high-performance liquid chromatographic assay for G418 sulfate (Geneticin). Antimicrob Agents Chemother 41 (1997) 661664. https://doi.org/10.1128/AAC.41.3.661.

[30] G. Suhren, K. Knappstein. Detection of incurred dihydrostreptomycin residues in milk by liquid chromatography and preliminary confirmation methods. Analyst 123 (1998) 2797-2801. https://doi.org/10.1039/a805050c.

[31] R. Oertel, V. Neumeister, W. Kirch. Hydrophilic interaction chromatography combined with tandemmass spectrometry to determine six aminoglycosides in serum. Journal of Chromatography A 1058 (2004) 197-201. https://doi.org/10.1016/i.chroma.2004.08.158.

[32] L. Zhang, J. Peng, J. Tang, B. Yuan, R. He, Y. Xiao. Description and validation of coupling high performance liquid chromatography with resonance Rayleigh scattering in aminoglycosides determination. Analytica chimica acta 706 (2011) 199-204. https://doi.org/10.1016/i.aca.2011.09.025.

[33] A.R. Breaud, C.L. Henemyre-Harris, S. Schools, N. Emezienna, W. Clarke. Rapid quantification of the aminoglycoside arbekacin in serum using high performance liquid chromatography-tandem mass spectrometry. J Clinica Chimica Acta 418 (2013) 102-106. https://doi.org/10.1016/i.cca.2013.01.004.

[34] J.B. Arsand, L. Jank, M.T. Martins, R.B. Hoff, F. Barreto, T.M. Pizzolato, C. Sirtori. Determination of aminoglycoside residues in milk and muscle based on a simple and fast extraction procedure followed by liquid chromatography coupled to tandem mass spectrometry and time of flight mass spectrometry. Talanta 154 (2016) 38-45. https://doi.org/10.1016/i.talanta.2016.03.045. 
[35] I.C. Roseboom, B. Thijssen, H. Rosing, J. Mbui, J.H. Beijnen, T.P. Dorlo. Highly sensitive UPLC-MS/MS method for the quantification of paromomycin in human plasma. Journal of pharmaceutical biomedical analysis 185 (2020) 113245. https://doi.org/10.1016/i.jpba.2020.113245.

[36] S.K. Maitra, T.T. Yoshikawa, J.-L. Hansen, I. Nilsson-Ehle, W. Palin, M.-C. Schotz, L. Guze. Serum gentamicin assay by high-performance liquid chromatography. J Clinical chemistry 23 (1977) 22752278. https://doi.org/10.1093/clinchem/23.12.2275.

[37] D.B. Haughey, D.M. Janicke, M. Adelman, J.J. Schentag. High-pressure liquid chromatography analysis and single-dose disposition of tobramycin in human volunteers. Antimicrob Agents Chemother 17 (1980) 649-653. https://doi.org/10.1128/aac.17.4.649.

[38] D.M. Barends, J.S. van der Sandt, A. Hulshoff. Micro determination of gentamicin in serum by highperformance liquid chromatography with ultraviolet detection. J Chromatogr 182 (1980) 201-210. https://doi.org/10.1016/s0378-4347(00)81624-2.

[39] N.-E. Larsen, K. Marinelli, A.M. Heilesen. Determination of gentamicin in serum using liquid column chromatography. Journal of Chromatography B: Biomedical Sciences 221 (1980) 182-187. https://doi.org/10.1016/S0378-4347(00)81023-3.

[40] S. Walker, P. Coates, Applications. High-performance liquid chromatographic method for determination of gentamicin in biological fluids. Journal of Chromatography B: Biomedical Sciences 223 (1981) 131-138. https://doi.org/10.1016/s0378-4347(00)80075-4.

[41] L.T. Wong, A.R. Beaubien, A.P. Pakuts, Applications. Determination of amikacin in microlitre quantities of biological fluids by high-performance liquid chromatography using 1-fluoro-2, 4dinitrobenzene derivatization. Journal of Chromatography B: Biomedical Sciences 231 (1982) 145154. https://doi.org/10.1016/s0378-4347(00)80518-6.

[42] J. D'Souza, R.I. Ogilvie. Determination of gentamicin components C1a, C2 and C1 in plasma and urine by high-performance liquid chromatography. J Chromatogr 232 (1982) 212-218. https://doi.org/10.1016/s0378-4347(00)86029-6.

[43] H. Kubo, T. Kinoshita, Y. Kobayashi, K. Tokunaga. Micro determination of gentamicin in serum by high-performance liquid chromatography. Journal of Chromatography B: Biomedical Sciences 227 (1982) 244-248. https://doi.org/10.1016/S0378-4347(00)81624-2.

[44] J. Marples, M. Oates. Serum gentamicin, netilmicin and tobramycin assays by high performance liquid chromatography. Journal of Antimicrobial Chemotherapy 10 (1982) 311-318. https://doi.org/10.1093Ljac/10.4.311.

[45] D. Barends, J. Blauw, M. Smits, A. Hulshoff. Determination of amikacin in serum by high-performance liquid chromatography with ultraviolet detection. Journal of Chromatography B: Biomedical Sciences Applications 276 (1983) 385-394. https://doi.org/10.1016/s0378-4347(00)85105-1.

[46] P.M. Kabra, P.K. Bhatnagar, M.A. Nelson, J.H. Wall, L.J. Marton. Liquid-chromatographic determination of tobramycin in serum with spectrophotometric detection. J Clinical chemistry 29 (1983) 672-674. https://doi.org/10.1093/jat/7.6.283.

[47] P.M. Kabra, P.K. Bhatnager, M.A. Nelson. Liquid chromatographic determination of amikacin in serum with spectrophotometric detection. Journal of Chromatography B: Biomedical Sciences 307 (1984) 224-229. https://doi.org/10.1016/S0378-4347(00)84093-1.

[48] H. Kubo, T. Kinoshita, Y. Kobayashi, K. Tokunaga. Micro-scale method for determination of tobramycin in serum using high-performance liquid chromatography. Journal of liquid chromatography 7 (1984) 2219-2228. https://doi.org/10.1080/01483918408068871.

[49] H. Kubo, Y. Kobayashi, T. Kinoshita. Fluorescence determination of streptomycin in serum by reversed-phase ion-pairing liquid chromatography. Analytical chemistry insights 58 (1986) 26532655. https://doi.org/10.1021/ac00126a017.

[50] G. Tamai, H. Imai, H. Yoshida. On-line deproteinization of serum sample for HPLC analysis of hydrophilic compounds and its application to gentamicin. Chromatographia 21 (1986) 519-522. https://doi.org/10.1007/BF02310539. 
[51] H. Kubo, H. Li, Y. Kobayashi, T. Kinoshita. Fluorometric determination of streptomycin in serum by high-performance liquid chromatography using mobile phase containing fluorogenic reagent. Analytical biochemistry 162 (1987) 219-223. https://doi.org/10.1016/0003-2697(87)90030-3.

[52] R. Rumble, M. Roberts. High-performance liquid chromatographic assay of the major components of gentamicin in serum. Journal of Chromatography B: Biomedical Sciences 419 (1987) 408-413. https://doi.org/10.1016/0378-4347(87)80308-0.

[53] T. Fujimoto, R. Tawa, S. Hirose. Fluorometric determination of sisomicin, an aminoglycoside antibiotic, in dried blood spots on filter paper by reversed-phase high-performance liquid chromatography with pre-column derivatization. Chemical pharmaceutical bulletin 36 (1988) 15711574. https://doi.org/10.1248/cpb.36.1571.

[54] H. Matsunaga, T. Fujimoto, R. Tawa, S. Hirose. An on-line cleanup procedure for large sample volume analysis of serum aminoglycoside antibiotics by reversed-phase high-performance liquid chromatography. Chemical J pharmaceutical Methods 36 (1988) 1565-1570. https://doi.org/10.1016/s0021-9673(98)00342-2.

[55] B. Wichert, H. Schreier, H. Derendorf. Sensitive liquid chromatography assay for the determination of amikacin in human plasma. Journal of pharmaceutical biomedical analysis 9 (1991) 251-254. https://doi.org/10.1016/0731-7085(91)80154-2.

[56] E.A. Papp, C.A. Knupp, R.H. Barbhaiya. High-performance liquid chromatographic assays for the quantification of amikacin in human plasma and urine. Journal of Chromatography B: Biomedical Sciences 574 (1992) 93-99. https://doi.org/10.1016/0378-4347(92)80102-V.

[57] F. Sar, P. Leroy, A. Nicolas, P. Archimbault, G. Ambroggi. Determination of amikacin in dog plasma by reversed-phase ion-pairing liquid chromatography with post-column derivatization. Analytical letters 25 (1992) 1235-1250. https://doi.org/10.1080/00032719208016125.

[58] M.A. Fennell, C.E. Uboh, R.W. Sweeney, L.R. Soma. Gentamicin in tissue and whole milk: an improved method for extraction and cleanup of samples for quantitation on HPLC. Journal of Agricultural Food Chemistry 43 (1995) 1849-1852. https://doi.org/10.1021/jf00055a018.

[59] M. Santos, E. Garcia, F. López, J. Lanao, A. Dominguez-Gil. Determination of netilmicin in plasma by HPLC. Journal of pharmaceutical biomedical analysis 13 (1995) 1059-1062. https://doi.org/10.1016/0731-7085(95)01331-E.

[60] D. Stead, R. Richards. Sensitive fluorimetric determination of gentamicin sulfate in biological matrices using solid-phase extraction, pre-column derivatization with 9-fluorenylmethyl chloroformate and reversed-phase high-performance liquid chromatography. Journal of Chromatography B: Biomedical Sciences 675 (1996) 295-302. https://doi.org/10.1016/0378-4347/95)00355-x.

[61] P.J. Kijak, J. Jackson, B. Shaikh. Determination of gentamicin in bovine milk using liquid chromatography with post-column derivatization and fluorescence detection. Journal of Chromatography B: Biomedical Sciences 691 (1997) 377-382. https://doi.org/10.1016/s03784347(96)00445-8.

[62] C.-c. Lin, E. Radwanski, C. Korduba, M. Affrime, M.N. Cayen. Pharmacokinetics of intramuscularly administered isepamicin in man. Chemotherapy 43 (1997) 86-93. https://doi.org/10.1159/000239541.

[63] D. Stead, R. Richards, Applications. Sensitive high-performance liquid chromatographic assay for aminoglycosides in biological matrices enables the direct estimation of bacterial drug uptake. Journal of Chromatography B: Biomedical Sciences 693 (1997) 415-421. https://doi.org/10.1016/s03784347(97)00032-7.

[64] N. Isoherranen, S. Soback. Determination of gentamicins C1, C1a, and C2 in plasma and urine by HPLC. J Clinical chemistry 46 (2000) 837-842. https://doi.org/10.1093/clinchem/46.6.837.

[65] C. Feng, S. Lin, H. Wu, S. Chen. Trace analysis of amikacin in human plasma by high-performance liquid chromatography. Chromatographia 53 (2001) S213-S217. https://doi.org/10.1007/BF024$\underline{90330 .}$. 
[66] A.I. Al-Amoud, B.J. Clark, H. Chrystyn. Determination of gentamicin in urine samples after inhalation by reversed-phase high-performance liquid chromatography using pre-column derivatisation with ophthalaldehyde. Journal of Chromatography B 769 (2002) 89-95. https://doi.org/10.1016/S15700232(01)00636-5.

[67] C.-H. Feng, S.-J. Lin, H.-L. Wu, S.-H. Chen. Trace analysis of tobramycin in human plasma by derivatization and high-performance liquid chromatography with ultraviolet detection. Journal of Chromatography B 780 (2002) 349-354. https://doi.org/10.1016/s1570-0232(02)00544-5.

[68] B.G. Keevil, S.J. Lockhart, D.P. Cooper. Determination of tobramycin in serum using liquid chromatography-tandem mass spectrometry and comparison with a fluorescence polarisation assay. Journal of Chromatography B 794 (2003) 329-335. https://doi.org/10.1016/S1570-0232(03)00492-6.

[69] R. Oertel, U. Renner, W. Kirch. Determination of neomycin by LC-tandem mass spectrometry using hydrophilic interaction chromatography. Journal of pharmaceutical biomedical analysis 35 (2004) 633-638. https://doi.org/10.1016/i.jpba.2004.01.018.

[70] N.C. Megoulas, M.A. Koupparis. Development and validation of a novel HPLC/ELSD method for the direct determination of tobramycin in pharmaceuticals, plasma, and urine. Anal Bioanal Chem $\mathbf{3 8 2}$ (2005) 290-296. https://doi.org/10.1007/s00216-004-2948-8.

[71] J.M. Serrano, M. Silva. Determination of amikacin in body fluid by high-performance liquidchromatography with chemiluminescence detection. Journal of Chromatography B 843 (2006) 20-24. https://doi.org/10.1016/j.jchromb.2006.05.016.

[72] M. Cherlet, S. De Baere, P. De Backer. Quantitative determination of dihydrostreptomycin in bovine tissues and milk by liquid chromatography-electrospray ionization-tandem mass spectrometry. Journal of mass spectrometry 42 (2007) 647-656. https://doi.org/10.1002/jms.1194.

[73] D.G. Mascher, C.P. Unger, H. Mascher. Determination of neomycin and bacitracin in human or rabbit serum by HPLC-MS/MS. Journal of pharmaceutical biomedical analysis 43 (2007) 691-700. https://doi.org/10.1016/j.jpba.2006.08.008.

[74] A.A. Al-Majed. A new LC method for determination of some aminoglycoside antibiotics in dosage forms and human plasma using 7-fluoro-4-nitrobenz-2-oxa-1, 3-diazole as a fluorogenic pre-column label. Chromatographia 68 (2008) 927-934. https://doi.org/10.1365/s10337-008-0792-2.

[75] M.E. Attema-de Jonge, J.M. Bekkers, H.M. Oudemans-van Straaten, R.W. Sparidans, E.J. Franssen. Simple and sensitive method for quantification of low tobramycin concentrations in human plasma using HPLC-MS/MS. Journal of Chromatography B 862 (2008) 257-262. https://doi.org/10.1016Li.jchromb.2007.12.008.

[76] S. Hosokawa, K. Nakamura, Y. Fujita, R. Horiuchi, K. Yamamoto. Determination of isepamicin in human plasma by HPLC with fluorescence detection after derivatization using 6-aminoquinolyl-Nhydroxysuccinimidyl-carbamate. Pharmaceutical Methods 31 (2008) 1866-1869. https://doi.org/10.1248/bpb.31.1866.

[77] M. Mashat, H. Chrystyn, B. Clark, K. Assi. Development and validation of HPLC method for the determination of tobramycin in urine samples post-inhalation using pre-column derivatisation with fluorescein isothiocyanate. Journal of Chromatography B 869 (2008) 59-66. https://doi.org/10.1016Li.jchromb.2008.05.012.

[78] X. Chang, J. Peng. LC Analysis of Isepamicin in Plasma Samples Post-Inhalation with Fluorescence Detection and Its Application to a Pharmacokinetic Study. Chromatographia 70 (2009) 1429-1433. https://doi.org/10.1365/s10337-009-1331-5.

[79] L. Baietto, A. D’Avolio, F.G. De Rosa, S. Garazzino, M. Michelazzo, G. Ventimiglia, M. Siccardi, M. Simiele, M. Sciandra, G. Di Perri. Development and validation of a simultaneous extraction procedure for HPLC-MS quantification of daptomycin, amikacin, gentamicin, and rifampicin in human plasma. Analytical bioanalytical chemistry 396 (2010) 791-798. https://doi.org/10.1007/s00216-009-3263-1.

[80] A.A. El-Zaher, M.A. Mahrouse. Utility of experimental Design in pre-column Derivatization for the Analysis of Tobramycin by HPLC-Fluorescence Detection: Application to Ophthalmic solution and Human plasma. Analytical chemistry insights 8 (2013) ACI. S11612. https://doi.org/10.4137/ACI.S11612. 
[81] Y. Bijleveld, T. de Haan, J. Toersche, S. Jorjani, J. van der Lee, F. Groenendaal, P. Dijk, A. van Heijst, A.W. Gavilanes, R. de Jonge. A simple quantitative method analysing amikacin, gentamicin, and vancomycin levels in human newborn plasma using ion-pair liquid chromatography/tandem mass spectrometry and its applicability to a clinical study. Journal of Chromatography B 951 (2014) 110118. https://doi.org/10.1016/i.jchromb.2014.01.035.

[82] B. Chauhan, S. Jalalpure. Analysis of amikacin in human serum by UHPLC with fluorescence detector using chloro-formate reagent with glycine. Pharmaceutical Methods 7 (2016) 99-103. https://doi.org/10.5530/phm.2016.7.15.

[83] M. Zu, J. Jiang, H. Zhao, S. Zhang, Y. Yan, S. Qiu, S. Yuan, J. Han, Y. Zhang, W. Guo. Rapid analysis of neomycin in cochlear perilymph of guinea pigs using disposable SPE cartridges and high performance liquid chromatography-tandem mass spectrometry. Journal of Chromatography B 1093 (2018) 52-59. https://doi.org/10.1016/i.jchromb.2018.06.055.

[84] A.C.C. da Silva, L.d.L.F. Lizot, M.F. Bastiani, M.V. Antunes, N. Brucker, R. Linden. Ready for TDM: Simultaneous quantification of amikacin, vancomycin and creatinine in human plasma employing ultra-performance liquid chromatography-tandem mass spectrometry. Clinical biochemistry $\mathbf{7 0}$ (2019) 39-45. https://doi.org/10.1016/j.clinbiochem.2019.06.011.

[85] A.C.C. da Silva, L.d.L.F. Lizot, M.F. Bastiani, M.V. Antunes, N. Brucker, R. Linden. Dried plasma spots for therapeutic monitoring of amikacin: Validation of an UHPLC-MS/MS assay and pharmacokinetic application. Journal of pharmaceutical biomedical analysis 184 (2020) 113201. https://doi.org/10.1016/j.jpba.2020.113201.

[86] J.-P. Antignac, K. de Wasch, F. Monteau, H. De Brabander, F. Andre, B. Le Bizec. The ion suppression phenomenon in liquid chromatography-mass spectrometry and its consequences in the field of residue analysis. Analytica Chimica Acta 529 (2005) 129-136. https://doi.org/10.1016/i.aca.2004.$\underline{08.055}$.

[87] C. Ghosh, C.P. Shinde, B.S. Chakraborty. Influence of ionization source design on matrix effects during LC-ESI-MS/MS analysis. Journal of Chromatography B 893 (2012) 193-200. https://doi.org/10.1016/j.jchromb.2012.03.012.

[88] B. Matuszewski, M. Constanzer, C. Chavez-Eng. Strategies for the assessment of matrix effect in quantitative bioanalytical methods based on HPLC- MS/MS. Analytical chemistry insights 75 (2003) 3019-3030. https://doi.org/10.1021/ac020361s.

[89] P.J. Taylor. Matrix effects: the Achilles heel of quantitative high-performance liquid chromatographyelectrospray-tandem mass spectrometry. Clinical biochemistry 38 (2005) 328-334. https://doi.orgL10.1016/i.clinbiochem.2004.11.007.

[90] O.A. Ismaiel, T. Zhang, R.G. Jenkins, H.T. Karnes. Investigation of endogenous blood plasma phospholipids, cholesterol and glycerides that contribute to matrix effects in bioanalysis by liquid chromatography/mass spectrometry. Journal of Chromatography B 878 (2010) 3303-3316. https://doi.org/10.1016/i.jchromb.2010.10.012.

[91] J.L. Little, M.F. Wempe, C.M. Buchanan. Liquid chromatography-mass spectrometry/mass spectrometry method development for drug metabolism studies: examining lipid matrix ionization effects in plasma. Journal of Chromatography B 833 (2006) 219-230. https://doi.org/10.1016/j.ichromb.2006.02.011.

[92] M. Garcia. The effect of the mobile phase additives on sensitivity in the analysis of peptides and proteins by high-performance liquid chromatography-electrospray mass spectrometry. Journal of Chromatography B 825 (2005) 111-123. https://doi.org/10.1016/j.jchromb.2005.03.041.

[93] H. Mei, Y. Hsieh, C. Nardo, X. Xu, S. Wang, K. Ng, W.A. Korfmacher. Investigation of matrix effects in bioanalytical high-performance liquid chromatography/tandem mass spectrometric assays: application to drug discovery. Rapid Communications in Mass Spectrometry 17 (2003) 97-103. https://doi.org/10.1002/rcm.876.

[94] W. Li, W. Jian, Y. Fu. Basic Sample Preparation Techniques in LC-MS Bioanalysis: Protein Precipitation, Liquid-Liquid Extraction, and Solid-Phase Extraction. Sample Preparation in LC-MS Bioanalysis (2019) 1-30. https://doi.org/10.1002/9781119274315.ch1. 
[95] A. Posyniak, J. Zmudzki, J. Niedzielska. Sample preparation for residue determination of gentamicin and neomycin by liquid chromatography. Journal of Chromatography A 914 (2001) 59-66. https://doi.org/10.1016/S0021-9673(00)00980-8.

[96] V. Gupta, A.D.K.J. Jain, N. Gill, K. Guptan. Development and validation of HPLC method-a review. International research journal of pharmaceutical applied sciences 2 (2012) 17-25.

[97] K.F.M. Opuni, J.A. Boadu, S.K. Amponsah, C.A. Okai. High performance liquid chromatography: A versatile tool for assaying antiepileptic drugs in biological matrices. Journal of Chromatography $B$ 1179 (2021) 122750. https://doi.org/10.1016/i.jchromb.2021.122750.

[98] M. Charde, A. Welankiwar, J. Kumar. Method development by liquid chromatography with validation. International Journal of Pharmaceutical Chemistry 4 (2014) 57-61. https://doi.org/10.7439/ijpc.v4i1.65 .

[99] Y. Vidushi, B. Meenakshi. A review on HPLC method development and validation. Res J Life Sci, Bioinform, Pharm Chem Sci 2 (2017) 178. https://doi.org/10.26479/2017.0206.12.

[100] D. Kowalczuk, R. Pietraś, B. Paw, A. Czerkies. Applying liquid chromatography with fluorescence detection to determine gentamicin. Pol. J. Environ. Stud 19 (2010) 587-591.

[101] H. Itagaki. Fluorescence spectroscopy. Experimental Methods in Polymer Science, Academic Press, San Diego, CA (2000) 155-260.

[102] R. LoBrutto, Y. Kazakevich, Reversed-phase HPLC, HPLC for Pharmaceutical Scientists, Wiley Online Library2007, pp. 139-195.

[103] K.F.M. Opuni, M. Al-Majdoub, Y. Yefremova, R.F. El-Kased, C. Koy, M.O. Glocker. Mass spectrometric epitope mapping. Mass Spectrom Rev 37 (2018) 229-241. https://doi.org/10.1002/mas.21516.

[104] M. Chen, H.H. Cai, F. Yang, D. Lin, P.H. Yang, J. Cai. Highly sensitive detection of chromium (III) ions by resonance Rayleigh scattering enhanced by gold nanoparticles. Spectrochim Acta A Mol Biomol Spectrosc 118 (2014) 776-781. https://doi.org/10.1016/j.saa.2013.09.058.

[105] W.R. LaCourse, S.J. Modi. Microelectrode applications of pulsed electrochemical detection. Electroanalysis: An International Journal Devoted to Fundamental Practical Aspects of Electroanalysis 17 (2005) 1141-1152. https://doi.org/10.1002/elan.200403233.

[106] P.J. Vandeberg, D.C. Johnson. Pulsed electrochemical detection of cysteine, cystine, methionine, and glutathione at gold electrodes following their separation by liquid chromatography. Analytical Chemistry 65 (1993) 2713-2718. https://doi.org/10.1021/ac00068a002.

[107] S.M. Moosavi, S. Ghassabian. Linearity of calibration curves for analytical methods: A review of criteria for assessment of method reliability. Calibration Validation of Analytical Methods $-A$ Sampling of Current Approaches, IntechOpen Ltd.: London, UK (2018) 109-127.

[108] A.M.d. Almeida, M.M. Castel-Branco, A. Falcao. Linear regression for calibration lines revisited: weighting schemes for bioanalytical methods. Journal of Chromatography B 774 (2002) 215-222. https://doi.org/10.1016/S1570-0232(02)00244-1.

[109] L.C. Rodríguez, A.M.G. Campa Ta, C.J. Linares, M.R. Ceba. Estimation of performance characteristics of an analytical method using the data set of the calibration experiment. Analytical letters 26 (1993) 1243-1258. https://doi.org/10.1080/00032719308019900.

[110] G. Smith. European Medicines Agency guideline on bioanalytical method validation: what more is there to say? Bioanalysis 4 (2012) 865-868. https://doi.org/10.4155/bio.12.44.

[111] L.V. Sonawane, B.N. Poul, S.V. Usnale, P.V. Waghmare, L.H. Surwase. Bioanalytical method validation and its pharmaceutical application-a review. Pharm Anal Acta 5 (2014) 2. https://doi.org/10.4172/2153-2435.1000288.

[112] A. Dasgupta. Advances in antibiotic measurement. Adv Clin Chem 56 (2012) 75-104. https://doi.org/10.1016/b978-0-12-394317-0.00013-3.

[113] P. Noone. Sisomicin, netilmicin and dibekacin. A review of their antibacterial activity and therapeutic use. Drugs 27 (1984) 548-578. https://doi.org/10.2165/00003495-198427060-00003. 
[114] J.P. Hieber, J.D. Nelson. Reevaluation of kanamycin dosage in infants and children. Antimicrob Agents Chemother 9 (1976) 899-902. https://doi.org/10.1128/AAC.9.6.899.

[115] Drugs.com. Streptomycin Dosage., 2021.

[116] G.G. Duncan, C.F. Clancy, J.R. Wolgamot, B. Beidleman. NEOMYCIN: RESULTS OF CLINICAL USE IN TEN CASES. Journal of the American Medical Association 145 (1951) 75-80. https://doi.org/10.1001/jama.1951.02920200015005.

[117] M. Urata, Y. Narita, D. Kadowaki, K. Tanoue, I. Tashiro, M. Fukunaga, A. Shibata, Y. Yoshida, K. Soejima, S. Hirata. Interaction of arbekacin with dialysis membrane. Renal Replacement Therapy 2 (2016) 35. https://doi.org/10.1186/s41100-016-0045-z.

[118] E. Novak, C.A. Schlagel, L.A. LeZotte, R.T. Pfeifer. The tolerance of high dose intravenous spectinomycin therapy in man. J Clin Pharmacol 14 (1974) 442-447. https://doi.org/10.1002/j.15524604.1974.tb02326.x.

[119] K.M. Krause, A.W. Serio, T.R. Kane, L.E. Connolly. Aminoglycosides: an overview. Cold Spring Harbor perspectives in medicine 6 (2016) a027029. https://doi.org/10.1101/cshperspect.a027029.

[120] V. Alampanos, V. Samanidou. Current trends in green sample preparation before liquid chromatographic bioanalysis. Current Opinion in Green and Sustainable Chemistry 31 (2021) 100499. https://doi.org/10.1016/j.cogsc.2021.100499.

[121] V. Alampanos, V. Samanidou, I. Papadoyannis. Trends in sample preparation for the hplc determination of penicillins in biofluids. Journal of Applied Bioanalysis 5 (2019) 9-17. https://doi.org/10.17145/jab.19.003.

[122] K.D. Clark, C. Zhang, J.L. Anderson. Sample Preparation for Bioanalytical and Pharmaceutical Analysis. Anal Chem 88 (2016) 11262-11270. https://doi.org/10.1021/acs.analchem.6b02935.

(C2022 by the authors; licensee IAPC, Zagreb, Croatia. This article is an open-access article distributed under the terms and conditions of the Creative Commons Attribution license (http://creativecommons.org/licenses/by/3.0/) (cc) B EY 